Bulletin of the Institute of Mathematics

Academia Sinica (New Series)

Vol. 14 (2019), No. 3, pp. 295-329

DOI: 10.21915 /BIMAS.2019302

\title{
GENERIC PROPERTY AND CONJUGACY CLASSES OF HOMOGENEOUS BOREL SUBALGEBRAS OF RESTRICTED LIE ALGEBRAS OF CARTAN TYPE (I): TYPE $W$
}

\author{
BIN SHU
}

\begin{abstract}
School of Mathematical Sciences, East China Normal University, Shanghai 200241, China. E-mail: bshu@math.ecnu.edu.cn
\end{abstract}

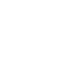

\begin{abstract}
Let $(\mathfrak{g},[p])$ be a finite-dimensional restricted Lie algebra over an algebraically closed field $\mathbb{K}$ of characteristic $p>0$, and $G$ be the adjoint group of $\mathfrak{g}$. We say that $\mathfrak{g}$ satisfies the generic property if $\mathfrak{g}$ admits generic tori introduced in [2]. In this paper, we first prove a generalized conjugacy theorem for Cartan subalgebras by means of the generic property. We then classify the $G$-conjugacy classes of homogeneous Borel subalgebras of the restricted simple Lie algebras $\mathfrak{g}=W(n)$ when $p>3$, and determine representatives of these classes. Here $W(n)$ is the so-called Jacobson-Witt algebra, by definition the derivation algebra of the truncated polynomial ring $\mathbb{K}\left[T_{1}, \cdots, T_{n}\right] /\left(T_{1}^{p}, \cdots, T_{n}^{p}\right)$. We also describe the closed connected solvable subgroups of $G$ associated with those representative Borel subalgebras.
\end{abstract}

\section{Introduction}

In the structure theory of classical Lie algebras (which arise from Lie algebras of connected semi-simple algebraic groups), Borel subalgebras (which arise from Lie algebras of Borel subgroups) along with Cartan subalgebras, Weyl groups, etc., are very important. These concepts are important tools in the structure and representation theory of classical Lie algebras (cf. 9], [8], [10] and [11] etc.). It is a basic fact that for a connected semi-simple

Received April 04, 2018.

AMS Subject Classification: 17B50, 17B05, 17B20.

Key words and phrases: Borel subalgebras of restricted Lie algebras, generic elements, generic property, Lie algebras of Cartan type.

This work is supported partially by the NSF of China (No. 11671138, 11771279), Shanghai Key Laboratory of PMMP (No. 13dz2260400). 
algebraic group $G$ and its Lie algebra $\mathfrak{g}$ the Borel (resp. Cartan) subalgebra of $\mathfrak{g}$ are conjugate under $G$. (Note that we can identify Borel subalgebras with maximal solvable subalgebras containing maximal tori when the ground field is an algebraically closed field of characteristic $\neq 2,3$ (cf. [8, $\S 14.3]$ and [18, §III.4]).) Especially, the variety $\mathbf{B}$ of all Borel subalgebras plays a key role for understanding the representations of $\mathfrak{g}$ (for example, the representation theory of $U_{\chi}(\mathfrak{g})$ for a nilpotent $p$-character $\chi$ is closely related to the geometry of the Springer fiber $\mathbf{B}_{\chi}$ which is a connected subvariety of $\mathbf{B}$, see [1]). By contrast, Cartan subalgebras and the maximal solvable subalgebras containing maximal tori, which will still be called Borel subalgebras in the present paper, of arbitrary restricted Lie algebras in prime characteristic, usually are no longer conjugate. In [5], [14], [15], Farnsteiner and Premet studied systematically Cartan subalgebras of restricted Lie algebras. Regular Cartan subalgebras which are the most important class of Cartan subalgebras, and which by definition are the ones containing a maximal torus of maximal dimension, are proved to be conjugate by means of a finite number of so-called elementary switchings (some invertible linear transformations provided by root vectors). Premet further proposed a conjecture on the conjugation of regular Cartan subalgebras under the adjoint group $G$ of $\mathfrak{g}$. As for Borel subalgebras, there is less study on them so far. Especially, for non-classical restricted simple Lie algebras, we neither know the number of conjugacy classes of Borel subalgebras nor what kind of role the Borel subalgebras play in the representation theory of these Lie algebras although their Cartan subalgebras are well-known (cf. [19]). Our motivation for writing this paper is to understand more about Borel subalgebras of non-classical restricted simple Lie algebras, and then possibly to develop the geometric aspects of non-classical Lie algebras and to apply them to representation theory.

The purpose of the present paper is twofold. One is to prove a generalized conjugacy theorem for Cartan subalgebras (Theorem 1.6), and by using this result to settle Premet's conjecture mentioned above in the negative (Remark 1.7); the other one is to study Borel subalgebras of restricted Lie algebras of type $W$ (Theorem 4.6).

According to the Block-Wilson-Strade-Premet classification of restricted simple Lie algebras over an algebraically closed field of characteristic $p>3$ (cf. [17]), it is known that aside from the analogues of the complex simple 
Lie algebras (called classical Lie algebras) there are usually four additional classes of restricted simple Lie algebras $\Perp$, the so-called restricted Lie algebras of Cartan type, among which the Jacobson-Witt algebras $W(n)$ will be a main topic of the present paper. In his article [16], Premet studied analogues of Weyl groups and of the Chevalley restriction theorem for complex simple Lie algebras, and the variety of nilpotent elements for $W(n)$. Along this direction, Bois, Farnsteiner and the author of the present paper developed some general theory of Weyl groups for restricted Lie algebras, and studied the Weyl groups for the other three classes of Cartan type Lie algebras (cf. 2] and [5]). They proposed in [2] the notion "generic tori" which play the same important role as the maximal tori in classical Lie algebras, associated with which Weyl groups and the Chevalley restriction theorem were obtained, with aid of the classification of isomorphism classes of maximal tori in [3] and [4]. Inspired by the work mentioned above, we initiate to study Borel subalgebras of restricted Lie algebras. One aim of the present paper is to determine the conjugacy classes of homogenous Borel subalgebras of $W(n)$ (see Definition 2.3 for the meaning of "homogeneous"). In particular, we consider generic Borel subalgebras and the associated solvable subgroups for $W(n)$. We will continue to finish the determination of conjugacy classes for Borel subalgebras of restricted simple Lie algebras of type $S(n)$ and $H(n)$ in the future. Along this direction the geometric structure of the variety $X$ of completely solvable homogeneous Borel subalgebras can be investigated (see [12]). (Recall that a Lie algebra $L$ is called completely solvable if $[L, L]$ is nilpotent.) There, the classification of conjugacy classes for completely solvable homogeneous Borel subalgebras turns out to be a subclassification of the one for homogeneous Borel subalgebras obtained in the present paper, which gives rise to a finite-length stratification of $X$ with a clear geometric description for each stratum.

Our paper is organized as follows. In the first section, we will give the notion generic property for a restricted Lie algebra $\mathfrak{g}$. Then we will prove that for generic Cartan subalgebras (see $§ 1.3$ for the definition), all of them are conjugate under the adjoint group of $\mathfrak{g}$ (Theorem 1.5). Consequently, we obtain a generalized conjugacy theorem for Cartan subalgebras (Theorem 1.6). The notion of generic Borel subalgebras is proposed here. In Section

\footnotetext{
${ }^{1}$ In the case $p=5$ there is one additional restricted simple Lie algebra, namely the restricted Melikian algebra.
} 
2 , we first recall some well-known results on $W(n)$. Then we propose a $\mathfrak{t}_{r^{-}}$ grading on $W(n)$ for any representative $\mathfrak{t}_{r}$ of the conjugacy classes of the maximal tori of $W(n)$. Finally, we introduce in this section the notion of homogeneous Borel subalgebras of $W(n)$. Section 3 is devoted to the study of standard Borel subspaces (whose definition will be seen in §3.1) and then to prove that those subspaces are Lie subalgebras and maximal solvable ones. In Section 4, we mainly make the argument on the classification of isomorphism classes of homogeneous Borel subalgebras of $W(n)$ (Theorem 4.6) when the characteristic $p$ of the ground filed $\mathbb{K}$ is greater than 3 . In Section 5 , we prove that the solvable groups associated with Borel subalgebras are connected, and further give a precise description of the solvable subgroups associated with the generic Borel subalgebras.

In this paper, we have to use many notations in the arguments. For the convenience of the reader we include a list of notation at the end.

\section{Generic property and Borel subalgebras}

Throughout, all vector spaces are assumed to be finite-dimensional and over an algebraically closed field $\mathbb{K}$ of prime characteristic $\operatorname{char}(\mathbb{K})=p>0$ unless mentioned otherwise. Given a restricted Lie algebra $(\mathfrak{g},[p])$, we have the adjoint group $G:=\operatorname{Aut}_{p}(\mathfrak{g})^{\circ}$, the identity component of the restricted automorphism group of $\mathfrak{g}$.

\subsection{Borel subalgebras}

A maximal solvable subalgebra $\mathcal{B}$ of a restricted Lie algebra $(\mathfrak{g},[p])$ is called a Borel subalgebra (or Borel for short) if $\mathcal{B}$ contains a maximal torus of $\mathfrak{g}$ (recall that a torus $\mathfrak{t}$ is by definition an abelian restricted subalgebra consisting of semi-simple elements, i.e., $X \in\left(X^{[p]}\right)_{p}$ for all $X \in \mathfrak{t}$, where $\left(X^{[p]}\right)_{p}$ denotes the restricted subalgebra generated by $X^{[p]}$ (see $\left.[20, \S 2.3]\right)$ ). A Cartan subalgebra is called regular if it contains a torus of maximal dimension.

The following observation is clear.

Lemma 1.1. Let $(\mathfrak{g},[p])$ be a restricted Lie algebra over $\mathbb{K}$. Then every maximal solvable subalgebra of $\mathfrak{g}$ is a restricted subalgebra. 
Proof. Let $\mathcal{B}$ be a given maximal solvable subalgebra of $\mathfrak{g}$. Note that the restricted subalgebra $(\mathcal{B})_{p}$ generated by $\mathcal{B}$ is still solvable. By the maximal solvability of Borel subalgebras, $\mathcal{B}$ coincides with $(\mathcal{B})_{p}$. Hence $\mathcal{B}$ itself is restricted.

\subsection{Generalized conjugacy of Cartan subalgebras}

For a restricted Lie algebra $(\mathfrak{g},[p])$, denote by $\mu(\mathfrak{g})$ the maximal dimension of all tori $\mathfrak{t} \subset \mathfrak{g}$. Following Premet, we say that $\mathfrak{h}$ is a regular Cartan subalgebra of $\mathfrak{g}$ if this Cartan subalgebra contains a torus of dimension $\mu(\mathfrak{g})$ (cf. [15]). The dimension of any regular Cartan subalgebra coincides with $r(\mathfrak{g})$, the rank of $\mathfrak{g}(\mathrm{cf}$. [14, Theorem 1]). For a given regular Cartan subalgebra $\mathfrak{h}$ with a maximal torus $\mathfrak{t}$, one has $\mathfrak{g}=\mathfrak{h} \oplus \sum_{\alpha \in \Delta(\mathfrak{g}, \mathfrak{h})} \mathfrak{g}_{\alpha}$, a root-space decomposition of $\mathfrak{g}$ with respect to $\mathfrak{h}$, where $\Delta(\mathfrak{g}, \mathfrak{h})$ is the corresponding root system. Then, any two regular Cartan subalgebras in $\mathfrak{g}$ can be obtained from each other by means of a finite number of elementary switchings defined via the root-space decompositions mentioned above (cf. [15, Theorem 1]). However, two such regular Cartan subalgebras are not necessarily conjugate under the $G$-action.

In [15, Conjecture 2], Premet proposed a conjecture that any finitedimensional restricted Lie algebra satisfies the following generalized conjugacy property for Cartan subalgebras:

Definition 1.2 (Generalized conjugacy of Cartan subalgebras). A restricted Lie algebra $(\mathfrak{g},[p])$ is said to admit generalized conjugacy of Cartan subalgebras if there exists a nonempty Zariski open subset $V(\subset \mathfrak{g})$ consisting of regular elements such that for any $u, v \in V$ the Cartan subalgebras $\mathfrak{g}^{0}(\operatorname{ad} u)$ and $\mathfrak{g}^{0}(\operatorname{ad} v)$ are conjugate under $G$, where the Fitting-nilspace is defined by $\mathfrak{g}^{0}(\operatorname{ad} X):=\left\{v \in \mathfrak{g} \mid \operatorname{ad} X^{m(X, v)} v=0\right.$ for some postive integer $\left.m(X, v)\right\}$ for $X \in \mathfrak{g}$.

\subsection{Generic property}

A torus $\mathfrak{t}_{\text {gen }}$ of $\operatorname{dim} \mathfrak{t}_{\text {gen }}=\mu(\mathfrak{g})$ is called generic if $G \cdot \mathfrak{t}_{\text {gen }}$ is a dense subset of $\bar{S}_{\mathfrak{g}}$, where $S_{\mathfrak{g}}$ stands for the Zariski closure of all semisimple elements in $\mathfrak{g}($ cf. $[2, \S 1])$. Set $\mathfrak{h}_{\text {gen }}:=C_{\mathfrak{g}}\left(\mathfrak{t}_{\text {gen }}\right)$, the centralizer of $\mathfrak{t}_{\text {gen }}$ in $\mathfrak{g}$. By 20 , Theorem 2.4.1], $\mathfrak{h}_{\text {gen }}$ is a regular Cartan subalgebra of $\mathfrak{g}$, which is called a 
generic Cartan subalgebra. A Borel subalgebra $\mathcal{B}$ of $\mathfrak{g}$ is called generic if it contains a generic torus of $\mathfrak{g}$.

Recall that $X \in \mathfrak{g}$ is called regular if $\mathfrak{g}^{0}(\operatorname{ad} X)$ has minimal dimension among all Fitting-nilspaces (cf. 14] and [15]). Denote by $\operatorname{Reg}(\mathfrak{g})$ the set of regular elements of $\mathfrak{g}$. Then $\operatorname{Reg}(\mathfrak{g})$ is an open dense subset of $\mathfrak{g}$.

A regular element $X$ in $\mathfrak{g}$ is called generic if $G \cdot \mathfrak{g}^{0}(\operatorname{ad} X)$ is a dense subset of $\mathfrak{g}$. It is worthwhile to remind the reader that it is not always the case that a restricted Lie algebra contains generic elements. (In view of Lemma 1.3 and Remark 1.4(1), the restricted contact Lie algebras are examples of such restricted Lie algebras.) From [2, Proposition 1.7] and the definitions, we can give a criterion for the existence of generic elements. We say that $\mathfrak{g}$ satisfies the generic property if $\mathfrak{g}$ admits generic tori.

Lemma 1.3. Let $(\mathfrak{g},[p])$ be a restricted Lie algebra. The generic property is equivalent to any one of the following items

(1) There are generic Borel subalgebras in $\mathfrak{g}$.

(2) There are generic Cartan subalgebras in $\mathfrak{g}$.

(3) There are generic elements in $\mathfrak{g}$.

\section{Remark 1.4.}

(1) For a classical Lie algebra $\mathfrak{g}$, all regular elements are generic because $G \cdot \mathfrak{h}$ is a dense subset of $\mathfrak{g}$ for any Cartan subalgebra $\mathfrak{h}$ (cf. 18, Theorem III 4.1]). According to [2, Proposition 3.3], restricted simple Lie algebras of type $W, S$ and $H$ have generic elements. So these Lie algebras satisfy the generic property. However, the restricted contact Lie algebras do not satisfy the generic property (cf. [2, Theorem 6.6]).

(2) Generic elements of $W(n), S(n)$ and $H(n)$ can be precisely described (cf. [13]).

If $\mathfrak{h}_{\text {gen }}$ is a generic Cartan subalgebra of $\mathfrak{g}$ containing the generic torus $\mathfrak{t}_{\text {gen }}$, then it follows from [5, Lemma 3.2] that there exists an open dense subset $U$ in $\mathfrak{t}_{\text {gen }}$ such that all elements of $U$ are generic. Furthermore, we will see in the forthcoming Theorem 1.6 that in such a case, there exists an open dense subset $V$ in $\mathfrak{g}$ such that all elements of $V$ are generic. 
Theorem 1.5. Let $(\mathfrak{g},[p])$ be a restricted Lie algebra satisfying the generic property. Then all generic Cartan subalgebras (resp. generic tori) are conjugate under $G$.

Proof. For any given two generic tori Cartan subalgebras $\mathfrak{h}_{1}$ and $\mathfrak{h}_{2}$, by definition $G \cdot \mathfrak{h}_{i}(i=1,2)$ are two dense subsets of $\mathfrak{g}$. Note that both $G \cdot \mathfrak{h}_{i}$ are constructible, thereby $G \cdot \mathfrak{h}_{i}(i=1,2)$ contain open dense subsets of $\mathfrak{g}$, respectively (cf. [6, II.§3] or $[9,4.4])$. On the other hand, the set $\operatorname{Reg}(\mathfrak{g})$ of regular elements of $\mathfrak{g}$ is also an open dense subset of $\mathfrak{g}$, which is $G$-stable. So we have that $\left(G \cdot \mathfrak{h}_{1}\right) \cap\left(G \cdot \mathfrak{h}_{2}\right) \cap \operatorname{Reg}(\mathfrak{g})$ is nonempty. Choose an element $H$ from this nonempty set. Then there is a unique regular Cartan subalgebra $\mathfrak{h}$ containing $H$. Hence $\mathfrak{h}$ coincides with $g_{1} \cdot \mathfrak{h}_{1}$ and $\mathfrak{g}_{2} \cdot \mathfrak{h}_{2}$ for some $g_{1}, g_{2} \in G$. Thus $\mathfrak{h}_{1}$ and $\mathfrak{h}_{2}$ are conjugate. This completes the proof.

\subsection{Generalized conjugacy theorem for Cartan subalgebras}

From the above theorem, we can prove the following generalized conjugacy theorem for Cartan subalgebras.

Theorem 1.6. Let $(\mathfrak{g},[p])$ be a restricted Lie algebra, and let $G$ be the adjoint group of $\mathfrak{g}$. Then the following statements are equivalent.

(1) $\mathfrak{g}$ admits generalized conjugacy of Cartan subalgebras;

(2) $\mathfrak{g}$ satisfies the generic property.

Proof. (2) $\Rightarrow(1)$ : Suppose that $\mathfrak{g}$ satisfies the generic property. Then $\mathfrak{g}$ has a generic Cartan subalgebra $\mathfrak{h}_{\text {gen }}$ (cf. Lemma 1.3). By definition, $G \cdot \mathfrak{h}_{\text {gen }}$ is dense in $\mathfrak{g}$, thereby a constructible subset of $\mathfrak{g}$. This means that $G \cdot \mathfrak{h}_{\text {gen }}$ contains an open dense subset $U$. Set $V:=U \cap \operatorname{Reg}(\mathfrak{g})$. Then $V$ is non-empty and an open dense subset of $\mathfrak{g}$. For any $v \in V, \mathfrak{g}^{0}(\operatorname{ad} v)$ is $G$ conjugate to $\mathfrak{g}^{0}\left(\operatorname{ad} v_{0}\right)$ for $v_{0} \in \mathfrak{h}_{\text {gen }} \cap \operatorname{Reg}(\mathfrak{g})$ with $v=g \cdot v_{0}$ for $g \in G$. From the uniqueness of the regular Cartan subalgebra containing $v_{0}$ it follows that $\mathfrak{g}^{0}\left(\operatorname{ad} v_{0}\right)$ coincides with $\mathfrak{h}_{\text {gen }}$. Thus $\mathfrak{g}^{0}(\operatorname{ad} v)$ is also a generic Cartan subalgebra. Thanks to Theorem 1.5, the Cartan subalgebras $\mathfrak{g}^{0}(\operatorname{ad} v)$ and $\mathfrak{g}^{0}(\operatorname{ad} u)$ are conjugate under $G$ for $u, v \in V$, and Statement (1) follows.

$(1) \Rightarrow(2)$ : Suppose that $V$ is a nonempty Zariski open subset of $\mathfrak{g}$ consisting of regular elements, and that the regular Cartan subalgebras $\mathfrak{g}^{0}(\operatorname{ad} v)$ for 
all $v \in V$ are conjugate under $G$. Note that $v \in \mathfrak{g}^{0}(\operatorname{ad} v)$. Hence for any given $v \in V, \overline{G \cdot \mathfrak{g}^{0}(\operatorname{ad} v)} \supset \bar{V}=\mathfrak{g}$. Thus, the Cartan subalgebra $\mathfrak{h}:=\mathfrak{g}^{0}(\operatorname{ad} v)$ is generic (in particular, $v$ is a generic element of $\mathfrak{g}$ ), and Statement (2) follows.

Remark 1.7. The above theorem shows that the statement in Premet's conjecture for a given restricted Lie algebra $\mathfrak{g}$ is valid if and only if $\mathfrak{g}$ satisfies the generic property. As mentioned in Example 1.4, restricted simple Lie algebras of type $W, S$ and $H$ satisfy the generic property. Hence the statement in Premet's conjecture holds for them. However, the restricted contact simple Lie algebras do not satisfy the generic property. So the contact restricted simple Lie algebras are examples showing the failure of Premet's conjecture? ${ }^{2}$.

In view of Theorem 1.5, we propose the following question.

Question 1.8. Let $(\mathfrak{g},[p])$ be a restricted Lie algebra satisfying the generic property. When are all generic Borel subalgebras conjugate under the adjoint group $G$ ?

In view of the known examples, we hope that for a restricted Lie algebra $\mathfrak{g}$ satisfying the generic property, all generic Borel subalgebras of maximal dimension are conjugate under $G$.

\section{Automorphisms and Standard Maximal Tori of the Restricted Simple Lie Algebra $W(n)$}

From now on, we assume that $\mathbb{K}$ is an algebraically closed field of characteristic $p \geqslant 3$. Set $\mathbb{P}=\{0,1, \ldots, p-1\}$. For an element $\mathbf{a}=\left(a_{1}, \ldots, a_{n}\right) \in$ $\mathbb{Z}^{n}$, we denote $|\mathbf{a}|:=a_{1}+\cdots+a_{n}$.

2.1. Define the truncated polynomial algebra $A(n)$ to be the quotient of the polynomial algebra $\mathbb{K}\left[T_{1}, \ldots, T_{n}\right]$ by the ideal generated by $T_{1}^{p}, \ldots, T_{n}^{p}$. Set $x_{i}$ to be the image of $T_{i}$ in the quotient. Then $A(n)=\sum_{\mathbf{a} \in \mathbb{P}^{n}} \mathbb{K} \mathbf{x}^{\mathbf{a}}$, where $\mathbf{x}^{\mathbf{a}}=x_{1}^{a_{1}} \cdots x_{n}^{a_{n}}$ with $\mathbf{a}=\left(a_{1}, \ldots, a_{n}\right) \in \mathbb{P}^{n}$. We sometimes write

\footnotetext{
${ }^{2}$ I was told that Skryabin had been aware of the failure of Premet's conjecture after the present work was finished and submitted.
} 
$A(n)$ as $\mathbb{K}\left[x_{1}, \ldots, x_{n}\right]$ to emphasize those indeterminants, and naturally regard $A(n)=\bigoplus_{i=0}^{n(p-1)} A_{[i]}$ as a $\mathbb{Z}$-graded algebra, where $A_{[i]}$ is spanned by monomials $\mathbf{x}^{\mathbf{a}}=x_{1}^{a_{1}} \cdots x_{n}^{a_{n}},|\mathbf{a}|=i$. The Jacobson-Witt algebra $W(n)$ is defined to be the derivation algebra of $A(n)$. This is to say, $W(n)$ consists of all linear transformations $D$ of $A(n)$ satisfying $D(f g)=D(f) g+f D(g)$ for $f, g \in A(n)$. It is easily seen that $W(n)$ is a free $A(n)$-module of rank $n$ with basis $\partial_{i}, i=1, \ldots, n$,

$$
W(n)=\sum_{i=1}^{n} A(n) \partial_{i}
$$

Here $\partial_{i}$ is the image of $\frac{\partial}{\partial T_{i}}$ in the quotient of the $\mathbb{K}\left[T_{1}, \ldots, T_{n}\right]$-module $\operatorname{Der}\left(\mathbb{K}\left[T_{1}, \ldots, T_{n}\right]\right)$ by the submodule arising from the ideal generated by $T_{i}^{p}, i=1, \ldots, n$. Hence

$$
\partial_{i} \mathbf{x}^{\mathbf{a}}=a_{i} \mathbf{x}^{\mathbf{a}-\epsilon_{i}}
$$

where $\epsilon_{i}=\left(\delta_{i, 1}, \ldots, \delta_{i, n}\right) \in \mathbb{P}^{n}, \delta_{i, j}=1$ if $i=j$, and $\delta_{i, j}=0$ otherwise.

Set $\mathfrak{g}=W(n)$. Then $\mathfrak{g}$ is a $\mathbb{Z}$-graded restricted simple Lie algebra. The $\mathbb{Z}$-grading of $W(n)$ arises from the one of the polynomial ring $\mathbb{K}\left[T_{1}, \ldots, T_{n}\right]$. More precisely, $\mathfrak{g}=\sum_{i} \mathfrak{g}_{[i]}$ with $\mathfrak{g}_{[i]}=\sum_{j=1}^{n} A_{[i+1]} \partial_{j}$ and the following properties hold:

$$
\mathfrak{g}=\bigoplus_{i=-1}^{h} \mathfrak{g}_{[i]},\left[\mathfrak{g}_{[i]}, \mathfrak{g}_{[j]}\right] \subset \mathfrak{g}_{[i+j]}, \mathfrak{g}_{[i]}^{[p]} \subset \mathfrak{g}_{[p i]}
$$

where $h=n(p-1)-1$, and we set $\mathfrak{g}_{[i]}:=0$ if $i$ is not between -1 and $h$. Associated with such a grading, one has a filtration

$$
\mathfrak{g}=\mathfrak{g}_{-1} \supset \mathfrak{g}_{0} \supset \cdots \supset \mathfrak{g}_{h} \supset 0
$$

for $\mathfrak{g}_{i}=\sum_{j=i}^{h} \mathfrak{g}_{[j]}, i=-1,0,1, \ldots, h$. For more details on $W(n)$, the reader is referred to [20, Ch.4].

\subsection{Automorphisms of $W(n)$}

Recall that an automorphism $\varphi \in \operatorname{Aut}(A(n))$ induces an automorphism $\bar{\varphi}$ of $W(n)$ defined via $\bar{\varphi}: D \mapsto \varphi \circ D \circ \varphi^{-1}$. The induced correspondence 
gives rise to a group isomorphism from $\operatorname{Aut}(A(n))$ to $\operatorname{Aut}(W(n))$ (cf. 21]). As to $\operatorname{Aut}(A(n))$, any element is determined by its action on the generators $x_{i}, i=1, \ldots, n$. We have a criterion that an algebra endomorphism $\varphi$ of $A(n)$ is an automorphism if and only if $\varphi$ stabilizes the unique maximal ideal of $A(n)$ and the determinant $\operatorname{det}\left(\left(\partial_{i} \varphi\left(x_{j}\right)\right)_{n \times n}\right)$ is invertible in $A(n)$.

Theorem 2.1 (21]). Let $\mathfrak{g}=W(n)$ over $\mathbb{K}$ of characteristic $p \geqslant 3$ (unless $n=1$ when $p>3)$. The following statements hold.

(1) The automorphism group Aut $(\mathfrak{g})$ coincides with the adjoint group $G=$ $A u t_{p}(\mathfrak{g})^{\circ}$ (see the paragraph before $\S 1.1$ for notation).

(2) The filtration (2.2) is G-invariant.

(3) The group $G$ is a semi-direct product $G=G_{0} \ltimes U$, where $G_{0} \cong G L(n, \mathbb{K})$ consists of those automorphisms preserving the $\mathbb{Z}$-grading of $\mathfrak{g}$, and

$$
U=\left\{g \in G ;\left(g-i d_{\mathfrak{g}}\right)\left(\mathfrak{g}_{i}\right) \subset \mathfrak{g}_{i+1}\right\}
$$

\subsection{Conjugacy classes of maximal tori}

According to Demuškin's result [3], we have the following conjugacy property for maximal tori of $W(n)$.

Theorem 2.2. Let $\mathfrak{g}=W(n)$. Then the following statements hold.

(1) Two maximal tori $\mathfrak{t}, \mathfrak{t}^{\prime}$ belong to the same $G$-orbit if and only if $\operatorname{dim} \mathfrak{t} \cap$ $\mathfrak{g}_{0}=\operatorname{dim} \mathfrak{t}^{\prime} \cap \mathfrak{g}_{0}$.

(2) There are $(n+1)$ conjugacy classes for the maximal tori of $\mathfrak{g}$. Each maximal torus of $\mathfrak{g}$ is conjugate to one of

$$
\mathfrak{t}_{r}=\sum_{i=1}^{n} \mathbb{K} z_{i} \partial_{i}, r=0,1, \ldots, n
$$

where $z_{i}=x_{i}$ for $i=1, \ldots, n-r$, and $z_{i}=1+x_{i}$ for $i=n-r+1, \ldots, n$.

We call these $\mathfrak{t}_{r}$ the standard maximal tori of $W(n)$.

\subsection{Gradings associated with $\mathfrak{t}_{r}$}

Note that the truncated polynomial algebra $A(n)$ can be presented as the quotient algebra $\mathbb{K}\left[T_{1}, \ldots, T_{n}\right] /\left(T_{1}^{p}-1, \ldots, T_{n}^{p}-1\right)$. Denote the image 
$T_{i}$ in the quotient algebra by $y_{i}$. Then we can write $A(n)$ as $\mathbb{K}\left[y_{1}, \ldots, y_{n}\right]$. Comparing with the notation used in $\S 2.1$, we actually have $y_{i}=1+x_{i}, i=$ $1, \ldots, n$. More generally, $A(n)$ can be presented as a truncated polynomial algebra

$$
\mathbb{K}\left[z_{1}, \ldots, z_{n-r} ; z_{n-r+1}, \ldots, z_{n}\right]
$$

with generators $z_{i}:=x_{i}, z_{j}:=y_{j}, i=1, \ldots, n-r ; j=n-r+1, \ldots, n$, and defining relations:

$$
\left[x_{i}, x_{i^{\prime}}\right]=\left[y_{j}, y_{j^{\prime}}\right]=\left[x_{i}, y_{j}\right]=x_{i}^{p}=y_{j}^{p}-1=0 .
$$

Thus, $W(n)$ can be presented, as a vector space,

$$
W(n)=\sum_{i=1}^{n} \sum_{\mathbf{c}(i) \in \mathbb{P}^{n}} \mathbb{K}^{\mathbf{c}(i)} \partial_{i},
$$

where $\mathbf{z}=\left(z_{1}, \ldots, z_{n}\right)$ and $\mathbf{z}^{\mathbf{c}(i)}=z_{1}^{c_{1}} \cdots z_{n}^{c_{n}}$ with $\mathbf{c}(i)=\left(c_{1}, \ldots, c_{n}\right) \in \mathbb{P}^{n}$. Associated to the presentation (2.3), there is a $\mathbb{Z}$-graded structure as follows, called $\mathbb{Z}\left(\mathfrak{t}_{r}\right)$-grading:

$$
\begin{aligned}
& W(n)=\bigoplus_{s} W_{[s]}^{\left(\mathfrak{t}_{r}\right)}, \text { with } \\
& W_{[s]}^{\left(\mathfrak{t}_{r}\right)}=\mathbb{K}-\operatorname{Span}\left\{\mathbf{z}^{\mathbf{c}(i)} \partial_{i}|| \mathbf{c}(i) \mid=s+1, i=1, \ldots, n\right\} .
\end{aligned}
$$

Actually, every homogenous space $W_{[s]}^{\left(\mathfrak{t}_{r}\right)}$ is a $\mathfrak{t}_{r}$-module. For the case $r=0$, the associated graded structure in (2.4) is called a standard-graded structure, coinciding with the one in (2.1). It is worthwhile to mention that for a given $v \in W(n), v$ belongs to $W_{[s]}^{\left(\mathrm{t}_{r}\right)}$ if and only if

$$
\operatorname{ad} T_{r}(v)=s v
$$

with $T_{r}:=\sum_{i=1}^{n} z_{i} \partial_{i}$, where $z_{i}=x_{i}$ and $z_{j}=1+x_{j}, i=1, \ldots, n-r$; $j=n-r+1, \ldots, n$.

When talking about $\mathfrak{t}_{0}$, we will omit the superscript for the associated graded structure as below

$$
W(n)=\bigoplus_{s} W_{[s]}, \text { with } W_{[s]}=\mathbb{K}-\operatorname{Span}\left\{\mathbf{x}^{\mathbf{c}(i)} \partial_{i}|| \mathbf{c}(i) \mid=s+1, i=1, \ldots, n\right\} .
$$


This gives rise to a $\mathbb{Z}$-graded Lie algebra structure for $W(n)$ as shown in (2.1). Thanks to Theorem 2.1, the associated filtration is invariant under $\operatorname{Aut}(W(n))$. Let $\mathfrak{H}$ be a subalgebra of $W(n)$. Call $\mathfrak{H}$ a $\mathbb{Z}\left(\mathfrak{t}_{r}\right)$-graded subalgebra if $\mathfrak{H}=\sum_{i} \mathfrak{H}_{[i]}^{\left(\mathfrak{t}_{r}\right)}$, where $\mathfrak{H}_{[i]}^{\left(\mathfrak{t}_{r}\right)}=\mathfrak{H} \cap W(n)_{[i]}^{\left(\mathfrak{t}_{r}\right)}$.

It will be useful to refine the grading of any $\mathbb{Z}\left(\mathfrak{t}_{r}\right)$-graded subalgebra of $W(n)$. If a subalgebra $\mathfrak{H}$ is $\mathbb{Z}\left(\mathfrak{t}_{r}\right)$-graded, we set for $\alpha \in \mathbb{P}^{n}$ with $|\alpha|=i+1$, $\mathfrak{H}_{\alpha}=\left\{v \in \mathfrak{H}_{[i]}^{\left(\mathfrak{t}_{r}\right)} \mid \operatorname{ad} z_{i} \partial_{i}(v)=\alpha_{i} v\right\}$. Then

$$
\mathfrak{H}=\sum_{\alpha \in \mathbb{P}^{n}} \mathfrak{H}_{\alpha}^{\left(\mathfrak{t}_{r}\right)}
$$

If $\mathfrak{H}$ has a decomposition as in (2.5), we say that $\mathfrak{H}$ is $\mathfrak{t}_{r}$-graded. The weight system of $\mathfrak{H}$ associated with $\mathfrak{t}_{r}$ is the set of all $\alpha$ with nonzero weight space $\mathfrak{H}_{\alpha}^{\left(\mathfrak{t}_{r}\right)}$. Naturally, the conditions of being $\mathfrak{t}_{r}$-graded and of being $\mathbb{Z}\left(\mathfrak{t}_{r}\right)$-graded are equivalent.

Definition 2.3. A subalgebra $Q$ is called homogeneous if its image under $\varphi$ is $\mathfrak{t}_{r}$-graded as long as $Q$ contains a maximal torus conjugate to $\mathfrak{t}_{r}$ under $\varphi \in \operatorname{Aut}(W(n))$.

2.5. Recall $W(n)_{[0]} \cong \mathfrak{g l}(n, \mathbb{K})$ under the mapping $x_{i} \partial_{j} \mapsto E_{i j}$, where $E_{i j}$ is the elementary matrix with all entries equal to 0 except the $(i, j)^{\text {th }}$-entry being equal to 1 . We then have a triangular decomposition $W(n)_{[0]}=$ $\sum_{i<j} \mathbb{K} x_{j} \partial_{i}+\mathfrak{t}_{0}+\sum_{i<j} \mathbb{K} x_{i} \partial_{j}$. Denote $\mathfrak{b}=\mathfrak{t}_{0}+\sum_{i<j} \mathbb{K} x_{i} \partial_{j}$, which is a standard Borel subalgebra of $W(n)_{[0]}$. We identify $W(n)_{[0]}$ with $\mathfrak{g l}(n, \mathbb{K})$ and $\operatorname{Aut}\left(W(n)_{[0]}\right)$ with $\operatorname{GL}(n, \mathbb{K})$ in the sequel whenever the context is clear.

Lemma 2.4. Assume the characteristic $p$ of the ground field $\mathbb{K}$ is greater than 3 , and $W(n)_{0}=\sum_{i \geqslant 0} W(n)_{[i]}$. The following statements hold.

(1) All maximal tori in $W(n)_{0}$ are conjugate to $\mathfrak{t}_{0}$ under Aut $(W(n))$.

(2) All Borel subalgebras of $W(n)_{0}$ are conjugate to $B_{0}:=\mathfrak{b}+W(n)_{1}$ under Aut $(W(n))$, where $W(n)_{1}=\sum_{i \geqslant 1} W(n)_{[i]}$.

Proof. (1) Set $\mathfrak{g}=W(n)$ and $G=\operatorname{Aut}(W(n))$. Denote the standard-graded and filtered structure by $\mathfrak{g}=\sum_{i} \mathfrak{g}_{[i]}$ and $\left\{\mathfrak{g}_{i}\right\}$ respectively. According to Demuškin's result (cf. [3]), we only need to show that maximal tori of $\mathfrak{g}_{0}$ are also the ones of $\mathfrak{g}$. Suppose that $\mathfrak{t}$ is a maximal torus of $\mathfrak{g}_{0}$ with basis 
$Z_{i}, i=1, \ldots, m$. We can write $Z_{i}=T_{i}+V_{i}$ with $T_{i} \in \mathfrak{g}_{[0]}$ and $V_{i} \in \mathfrak{g}_{1}$. Note that $V_{i}$ is nilpotent. Hence $T_{i}$ must be a semisimple element. Thus the set $\left\{T_{i}, i=1, \ldots, m\right\}$ spans a maximal torus of $\mathfrak{g}_{0}$, thereby the maximal torus of $\mathfrak{g}_{[0]}$. The latter is conjugate to $\mathfrak{t}_{0}$ under $\operatorname{GL}(n, \mathbb{K})$ (cf. [18, Theorem III.4.1]. The first assertion is proved.

(2) We first observe that for any Borel subalgebra $\mathfrak{b}^{\prime}$ of $\mathfrak{g}_{[0]} \cong \mathfrak{g l}(n, \mathbb{K})$, $\mathcal{B}^{\prime}:=\mathfrak{b}^{\prime}+\mathfrak{g}_{1}$ must be a Borel subalgebra of $\mathfrak{g}_{0}$. The solvability of $\mathcal{B}^{\prime}$ comes from the fact that $\mathcal{B}^{(n)} \subset \mathfrak{g}_{1}$, and $\mathfrak{g}_{1}$ is nilpotent, where $L^{(m)}$ for a Lie algebra $L$ denotes the $m$-th derived ideal, i.e. $L^{(m)}:=\left[L^{(m-1)}, L^{(m-1)}\right]$ with $L^{(0)}:=L$. In order to check the maximal solvability of $\mathcal{B}^{\prime}$, we observe that $\mathcal{B}^{\prime} \subset \mathfrak{g}_{0}$ is standard-graded with $\mathcal{B}_{[0]}^{\prime}=\mathfrak{b}^{\prime}$ and $\mathcal{B}_{[i]}^{\prime}=\mathfrak{g}_{[i]}$ for all $i>0$. So any other solvable subalgebra of $\mathfrak{g}_{0}$ containing $\mathcal{B}^{\prime}$ is also standard-graded. Then the maximal solvability of $\mathfrak{b}^{\prime}$ in $\mathfrak{g}_{[0]}$ implies that of $\mathcal{B}^{\prime}$ in $\mathfrak{g}_{0}$. Note that any Borel subalgebra of $\mathfrak{g l}(n, \mathbb{K})$ is conjugate to the standard Borel subalgebra under $\operatorname{GL}(n, \mathbb{K}) \subset \operatorname{Aut}(W(n))$ (cf. [8, §14.3-4]). It follows that any Borel subalgebra of $\mathfrak{g}_{0}$, up to conjugation, can be constructed in this way.

By the invariance of the filtration $\left\{\mathfrak{g}_{i}\right\}$ under $G$ (Theorem 2.1), we know those Borel subalgebras are conjugate to $\mathrm{B}_{0}=\mathfrak{b}+W(n)_{1}$ under $\operatorname{GL}(n, \mathbb{K})$. This completes the proof.

\subsection{An application to $W(1)$}

We assume $\mathfrak{g}=W(1)$ and the characteristic $p$ of the ground field is greater than 3 . In this special case, we adopt the notations $x$ and $\partial$, with the same meaning as $x_{1}$ and $\partial_{1}$, respectively. It is easy to see that any subalgebra of $W(1)$ which contains $\mathfrak{t}_{i}(i \in\{0,1\})$ is $\mathfrak{t}_{i}$-graded. Hence, all Borel subalgebras of $W(1)$ are homogeneous, and therefore we have the following results for the conjugacy classes of Borel subalgebras of $W(1)$.

Proposition 2.5. There are only two conjugacy classes of Borel subalgebras for the Witt algebra $W(1)$. The standard representatives are $B_{1}:=\mathbb{K} \partial+\mathbb{K} x \partial$ and $B_{0}$ as defined in Lemma 2.4 .

Proof. Recall that $W(1)$ admits two conjugacy classes of maximal tori, with representatives $\mathfrak{t}_{0}=\mathbb{K} x \partial$, and $\mathfrak{t}_{1}=\mathbb{K}(1+x) \partial$. For a given Borel subalgebra $\mathcal{B}$, we can assume that up to conjugation, either $\mathcal{B} \supset \mathfrak{t}_{0}$, or $\mathcal{B} \supset \mathfrak{t}_{1}$. 
In the case when $\mathcal{B} \supset \mathfrak{t}_{0}$, the homogeneous property implies that $\mathcal{B}$ is standard-graded. So $\mathcal{B}=\sum_{i} \mathcal{B}_{[i]}$, where $\mathcal{B}_{[0]}=W_{[0]}$. If $\operatorname{dim} \mathcal{B}_{[-1]}=1$, the maximal solvable subalgebra $\mathcal{B}$ contains $\mathbb{K} \partial+\mathbb{K} x \partial$. However, the latter is already a maximal solvable subalgebra. This is because any subalgebra properly containing $\mathbb{K} \partial+\mathbb{K} x \partial$ must contain $\mathbb{K} \partial+\mathbb{K} x \partial+\mathbb{K} x^{2} \partial$, while the latter is not solvable. So $\mathcal{B}=\mathbb{K} \partial+\mathbb{K} x \partial$ in this case. If $\operatorname{dim} \mathcal{B}_{[-1]}=0$, then $\mathcal{B} \subset W(1)_{0}$. The latter is solvable. The maximal solvability of $\mathcal{B}$ implies $\mathcal{B}=\mathrm{B}_{0}$ (note that $\mathrm{B}_{0}$ coincides with $W(1)_{0}$ in the case of $W(1)$ ).

In the following argument, we assume that $\mathcal{B} \supset \mathfrak{t}_{1}$. By the homogeneous property again, $\mathcal{B}$ is $\mathfrak{t}_{1}$-graded. Consider the $\mathbb{Z}\left(\mathfrak{t}_{1}\right)$-graded structure $W(1)=$ $\bigoplus_{i} W(1)_{[i]}^{\left(\mathfrak{t}_{1}\right)}$. In the case that $\operatorname{dim} \mathcal{B}_{[-1]}^{\left(\mathrm{t}_{1}\right)}=1$, we have that $\mathcal{B} \supset \mathbb{K} \partial+\mathbb{K}(1+$ $x) \partial=\mathbb{K} \partial+\mathbb{K} x \partial=\mathrm{B}_{1}$. Hence $\mathcal{B}=\mathrm{B}_{1}$. Next we consider the remaining situation $\mathcal{B} \supset \mathfrak{t}_{1}$ and $\operatorname{dim} \mathcal{B}_{[-1]}^{\left(\mathfrak{t}_{1}\right)}=0$. In such a case, $\mathcal{B} \subset \mathbb{K}(1+x) \partial+$ $\sum_{i>0} \mathbb{K}(1+x)^{i} \partial$. As $\mathcal{B}$ is a maximal solvable subalgebra, $\mathcal{B} \supsetneqq \mathbb{K}(1+x) \partial$. Therefore, there exists $D:=(1+x)^{a} \partial \in \mathcal{B}$ with $p>a>1$ (note the homogeneous property). Consequently, one can conclude that $\mathcal{B}$ coincides with $\mathbb{K}(1+x) \partial+\mathbb{K}(1+x)^{a} \partial$ since the latter is readily known to be a maximal solvable subalgebra. Actually, any subalgebra properly containing $\mathbb{K}(1+$ $x) \partial+\mathbb{K}(1+x)^{a} \partial$ must contain $\mathbb{K}(1+x) \partial+\mathbb{K}(1+x)^{a} \partial+\mathbb{K} \partial$ (note that $\left.(1+x)^{p}=1\right)$, which contradicts the assumption $\operatorname{dim} \mathcal{B}_{[-1]}^{\left(\mathfrak{t}_{1}\right)}=0$. Next, we will show that $\mathcal{B}\left(=\mathbb{K}(1+x) \partial+\mathbb{K}(1+x)^{a} \partial\right)$ is conjugate to $\mathrm{B}_{1}$. Consider $\varphi \in$ $\operatorname{Aut}(W(1))$ which is defined via $\varphi(x)=(1+x)^{b}-1$, where $b \in\{2, \ldots, p-1\}$ with $(1-a) b \equiv 1 \bmod p$. Then the inverse $\varphi^{-1}: x \mapsto(1+x)^{p-a+1}-1$. We have an automorphism $\bar{\varphi}$ of $W(1)$ induced by $\varphi$ (see $\S 2.2$ ), denoted by $\Phi$. By a straightforward computation, we have

$$
\begin{aligned}
\Phi\left((1+x)^{a} \partial\right) & =(p-a+1) \partial, \\
\Phi((1+x) \partial) & =(p-a+1)(1+x) \partial .
\end{aligned}
$$

Under the conjugation via such a $\Phi$, we have $\mathcal{B} \cong \mathbb{K} \partial+\mathbb{K}(1+x) \partial=\mathrm{B}_{1}$.

Remark 2.6. For $\mathfrak{g}=W(1)$, we can show that any maximal solvable subalgebra is a Borel. Actually, a given maximal subalgebra $\mathcal{B}$ can be endowed with a filtration structure $\left\{\mathcal{B}_{i}\right\}$ inheriting the one from $\mathfrak{g}$ as in (2.2). Consider the graded subalgebra $\operatorname{Gr}(\mathcal{B})$ of $\mathfrak{g}$. Then $\operatorname{Gr}(\mathcal{B})=\sum_{i=-1}^{p-2} \operatorname{Gr}(\mathcal{B})_{[i]}$ is also a maximal solvable subalgebra of $\mathfrak{g}$. Now $\operatorname{Gr}(\mathcal{B})$ is normalized by $\mathbb{K} x \partial$. 
Hence, $\operatorname{Gr}(\mathcal{B})$ contains $\mathbb{K} x \partial$. This implies that $\mathcal{B}$ contains nonzero semisimple elements, and then contains some maximal torus of $\mathfrak{g}$. Therefore, the above proposition covers the main result of [22].

The above arguments of classifying Borels for $W(1)$ will be extended to the general case in the next sections. The ideas are essentially the same. The role of $B_{1}$ here will be replaced by an extreme Borel subalgebra $B_{n}$ which will be called the fully-switched Borel subalgebra in the general case (see $\S 3.1)$.

\section{Standard Borel Subalgebras}

We maintain the notations as before. Suppose $n>1$.

\subsection{Borel subspaces}

We will first introduce $(n+1)$ vector subspaces $\mathrm{B}_{q}$ in $W(n), q=0,1, \ldots, n$. Recall $\mathrm{B}_{0}=\mathfrak{b}+W(n)_{1}$ as defined in Lemma 2.4. Next, keeping the same notation as in (2.3) we set

$$
\mathrm{B}_{n}=W(n)_{[-1]}+\mathfrak{b}+\sum_{q=1}^{n} \sum_{\mathbf{a}(q)} \mathbb{K} \mathbf{x}^{\mathbf{a}(q)} \partial_{q},
$$

where $\mathbf{x}=\left(x_{1}, \ldots, x_{n}\right)$ and $\mathbf{a}(q):=\left(a_{1}, \ldots, a_{q}, 0, \ldots, 0\right) \in \mathbb{P}^{n}$ with $|\mathbf{a}(q)|>$ $1, a_{i}$ runs through $\mathbb{P}$ for $i=1, \ldots, q-1$, and $a_{q}=0,1$. We call $\mathrm{B}_{0}$ and $\mathrm{B}_{n}$ the unswitched Borel subspace and the fully-switched Borel subspace, respectively.

Before introducing general Borel subspaces $\mathrm{B}_{q}(0<q<n)$, we will need some conventions of which some have appeared before but now are introduced formally

Conventions 3.1. Let $z_{i}$ be either $x_{i}$ or $\left(1+x_{i}\right), i=1, \ldots, n$. For $a$ subsequence $\mathbf{u}=\left(u_{1}, \ldots, u_{q}\right)$ of the sequence $\left(z_{1}, \ldots, z_{n}\right)$, i.e, $\left\{u_{1}, \ldots, u_{q}\right\} \subset$ $\left\{z_{1}, \ldots, z_{n}\right\}$, we adopt the notations:

(1) Set $B_{0}\left(u_{1}, \ldots, u_{q}\right)$ and $B_{q}\left(u_{1}, \ldots, u_{q}\right)$ to be the unswitched Borel subspace, and the fully-switched Borel subspace of $W(q)$ respectively. Here 
$W(q)$ is the derivation algebra of $A(q)=\mathbb{K}\left[u_{1}, \ldots, u_{q}\right]$, which is the subalgebra of the truncated polynomial algebra $A(n)=\mathbb{K}\left[x_{1}, \ldots, x_{n}\right]$ generated by $u_{1}, \ldots, u_{q}$. For stressing the indeterminants, we sometimes will use the notations $A\left(u_{1}, \ldots, u_{q}\right)$ and $W\left(u_{1}, \ldots, u_{q}\right)$ for $A(q)$ and $W(q)$ respectively.

(2) Set $\mathfrak{t}_{0}\left(u_{1}, \ldots, u_{q}\right):=x_{i_{1}} \partial_{i_{1}}+\cdots+x_{i_{q}} \partial_{i_{q}}$, and $\mathfrak{t}_{q}\left(u_{1}, \ldots, u_{q}\right)=(1+$ $\left.x_{i_{1}}\right) \partial_{i_{1}}+\cdots+\left(1+x_{i_{q}}\right) \partial_{i_{q}}$ when $\left(u_{1}, \ldots, u_{q}\right)=\left(x_{i_{1}}, \ldots, x_{i_{q}}\right)$. Those $\mathfrak{t}_{0}$ and $\mathfrak{t}_{q}$ mean the first and last standard tori of $W\left(x_{i_{1}}, \ldots, x_{i_{q}}\right)$ respectively.

(3) Set $\mathbf{u}^{\mathbf{a}}:=u_{1}^{a_{1}} \cdots u_{q}^{a_{q}}$ if $\mathbf{a}=\left(a_{1}, \ldots, a_{q}\right) \in \mathbb{P}^{q}$.

Now we are in the position to define the $q$ th Borel subspace $\mathrm{B}_{q}(0<q<$ $n)$. Let us first take $\mathbf{u}=\left(x_{1}, \ldots, x_{n-q}\right)$ and $\mathbf{w}=\left(x_{n-q+1}, \ldots, x_{n}\right)$. Define

$$
\mathrm{B}_{q}=\mathrm{B}_{0}\left(x_{1}, \ldots, x_{n-q}\right)+Q_{q}+\mathrm{B}_{q}\left(x_{n-q+1}, \ldots, x_{n}\right),
$$

where

$$
Q_{q}=\sum_{i=1}^{n-q} \sum_{\mathbf{a}(i), \mathbf{b}(i)} \mathbb{K} \mathbf{u}^{\mathbf{a}(i)} \mathbf{w}^{\mathbf{b}(i)} \partial_{i}+\sum_{j=n-q+1}^{n} \sum_{\mathbf{a}(j) ; \mathbf{b}(j)} \mathbb{K} \mathbf{u}^{\mathbf{a}(j)} \mathbf{w}^{\mathbf{b}(j)} \partial_{j},
$$

where $\mathbf{a}(i):=\left(a_{1}, \ldots, a_{n-q}\right) \in \mathbb{P}^{n-q}$ is subject to the condition that either $|\mathbf{a}(i)|>1$ or $|\mathbf{a}(i)|=1=a_{1}+\cdots+a_{i}$, while $\mathbf{a}(j) \in \mathbb{P}^{n-q}$ is subject to the condition $|\mathbf{a}(j)|>0$, and $\mathbf{b}(-):=\left(b_{n-q+1}, \ldots, b_{n}\right)$ runs through $\mathbb{P}^{q}$ for $(-)=(i),(j)$, subject to the condition $|\mathbf{b}(i)|>0$.

In the subsequent subsections, we will prove that all $\mathrm{B}_{r}$ are Borel subalgebras of $W(n)(r=0,1, \ldots, n)$.

\subsection{Switched positive root systems of the rigid root system}

Set $E=\{-1,0\}$. Consider a subset $\Delta$ of $\Gamma:=\mathbb{P}^{n} \times E^{n}$ :

$$
\Delta=\left\{\left(a_{1}, \ldots, a_{n}\right) \times\left(\eta_{1}, \ldots, \eta_{n}\right) \in \Gamma \mid \eta_{1}+\cdots+\eta_{n}=-1\right\},
$$

which means that elements in $\Delta$ are of form $\left(\sum_{i} a_{i} \epsilon_{i}\right) \times\left(-\epsilon_{s}\right)$. Set

$$
\bar{\Delta}=\Delta \cup\{\infty\}
$$


For $\alpha=\left(\sum_{i} a_{i} \epsilon_{i}\right) \times\left(-\epsilon_{s}\right)$ and $\beta=\left(\sum_{i} b_{i} \epsilon_{i}\right) \times\left(-\epsilon_{t}\right) \in \Delta(s, t \in\{1,2, \ldots, n\})$, we define an ordered operator $\sharp: \bar{\Delta} \times \bar{\Delta} \rightarrow \bar{\Delta}$ by

$$
\alpha \sharp \beta:= \begin{cases}\left(\sum_{i}\left(a_{i}+b_{i}\right) \epsilon_{i}-\epsilon_{s}\right) \times\left(-\epsilon_{t}\right), & \text { if } a_{s}+b_{s} \neq 0 ; \\ \infty, & \text { otherwise; }\end{cases}
$$

and $\infty \sharp$ any $=$ any $\sharp \infty=\infty$. Here any means any element of $\Delta$. Then $W(n)$ can be decomposed into a direct sum of one-dimensional root spaces, associated with the so-called rigid root system as below:

$$
W(n)=\sum_{\alpha \in \bar{\Delta}} W(n)_{\alpha}
$$

where $W(n)_{\alpha}:=\mathbb{K} x_{1}^{a_{1}} \cdots x_{n}^{a_{n}} \partial_{j}$ for $\alpha=\left(a_{1}, \ldots, a_{n}\right) \times\left(-\epsilon_{j}\right)$, and $W(n)_{\infty}:=$ 0 . We call $\bar{\Delta}$ the rigid root system of $W(n)$. Then we have

$$
\left[W(n)_{\alpha}, W(n)_{\beta}\right] \subset W(n)_{\alpha \sharp \beta}+W(n)_{\beta \sharp \alpha} .
$$

Associated with the unswitched and fully-switched Borel subspaces, we define positive root systems $\bar{\Delta}(0)_{+}$, and $\bar{\Delta}(n)_{+}$, respectively, as below

$$
\begin{aligned}
& \bar{\Delta}(0)_{+}:=\left\{\epsilon_{i} \times\left(-\epsilon_{j}\right) \mid 1 \leqslant i \leqslant j \leqslant n\right\} \cup\{\infty\} \cup \\
&\left\{\sum_{i=1}^{n} a_{i} \epsilon_{i} \times\left(-\epsilon_{j}\right) \mid a_{1}+\cdots+a_{n}>1, j=1, \ldots, n\right\} ; \\
& \bar{\Delta}(n)_{+}:=\left\{0 \times\left(-\epsilon_{j}\right) \mid j=1, \ldots, n\right\} \cup\left\{\epsilon_{i} \times\left(-\epsilon_{j}\right) \mid 1 \leqslant i \leqslant j \leqslant n\right\} \cup\{\infty\} \cup \\
&\left\{\sum_{i=1}^{n} a_{i} \epsilon_{i} \times\left(-\epsilon_{j}\right) \mid a_{1}+\cdots+a_{n}=a_{1}+\cdots+a_{j}>1 ;\right. \\
&\left.a_{j}=0, \text { or } 1 ; j=1,2, \ldots, n\right\} .
\end{aligned}
$$

Then $\mathrm{B}_{0}=\sum_{\alpha \in \bar{\Delta}(0)_{+}} W(n)_{\alpha}$ and $\mathrm{B}_{n}=\sum_{\alpha \in \bar{\Delta}(n)_{+}} W(n)_{\alpha}$. Generally, we set

$$
\bar{\Delta}(q)_{+}=\bar{\Delta}^{\left(x_{1}, \ldots, x_{n-q}\right)}(0)_{+} \cup \bar{\Delta}^{\left(x_{n-q+1}, \ldots, x_{n}\right)}(q)_{+} \cup \bar{\Delta}\{Q\}_{+},
$$

where $\bar{\Delta}^{\left(x_{1}, \ldots, x_{n-q}\right)}(0)_{+}$, and $\bar{\Delta}^{\left(x_{n-q+1}, \ldots, x_{n}\right)}(q)_{+}$denote the unswitched positive root system and the fully-switched positive root system associated with $\mathbb{K}\left[x_{1}, \ldots, x_{n-q}\right]$ and $\mathbb{K}\left[x_{n-q+1}, \ldots, x_{n}\right]$, respectively; and $\bar{\Delta}\{Q\}_{+}$denotes a 
subset of $\bar{\Delta}_{+}$as below:

$$
\begin{aligned}
\bar{\Delta}\{Q\}_{+}= & \left\{\left(\sum_{i=1}^{n-q} a_{i} \epsilon_{i}+\sum_{j=n-q+1}^{n} b_{j} \epsilon_{j}\right) \times\left(-\epsilon_{r}\right) \mid 1 \leqslant r \leqslant n-q ;\right. \\
& \text { either } a_{1}+\cdots+a_{n-q}>1 \text { or } a_{1}+\cdots+a_{n-q}=a_{1}+\cdots+a_{r}=1 ; \\
& \left.b_{n-q+1}+\cdots+b_{n}>0\right\} \bigcup\{\infty\} \\
& \bigcup\left\{\left(\sum_{i=1}^{n-q} a_{i} \epsilon_{i}+\sum_{j=n-q+1}^{n} b_{j} \epsilon_{j}\right) \times\left(-\epsilon_{s}\right) \mid n-q+1 \leqslant s \leqslant n ;\right. \\
& \left.a_{1}+\cdots+a_{n-q}>0\right\} .
\end{aligned}
$$

By the above construction, we have

Lemma 3.2. Maintain the notations as above. The following statements hold.

(1) $B_{q}=\sum_{\alpha \in \bar{\Delta}(q)_{+}} W(n)_{\alpha}, q=0,1, \ldots, n$.

(2) $B_{q}$ contains the maximal tori $\mathfrak{t}_{r}, r=0,1, \ldots, q$.

(3) Furthermore, $B_{q}$ is a restricted $\mathfrak{t}_{r}$-module for $r=0,1, \ldots, q$. As a $\mathfrak{t}_{0}$ module the weight system is described as below, in the sense of (2.5):

$\left\{\mathbf{a}=\left(a_{1}, \ldots, a_{n}\right) \in \mathbb{P}^{n} \mid a_{i}\right.$ coincides with the ith entriy of $\bar{\alpha}$ for $\left.\alpha \in \Delta(q)_{+}\right\}$

where $\bar{\alpha}=(\cdot)+(\cdot \cdot)$ for $\alpha=(\cdot) \times(\cdot \cdot)$ and $\Delta(q)_{+}=\bar{\Delta}(q)_{+} \backslash\{\infty\}$.

(4) $B_{q}$ is a subalgebra of $W(n)$.

Proof. The first three statements are clear. In order to prove the last one, we only need to check that the operator $\sharp$ is closed on $\bar{\Delta}(q)_{+}$in view of the first three statements, and Formula (3.2), along with the homogenous condition. By a direct computation, we easily obtain that it is closed on $\bar{\Delta}(0)_{+}$and $\bar{\Delta}(n)_{+}$. For an arbitrary $q$, we note that in

$$
\mathrm{B}_{q}=\mathrm{B}_{0}\left(x_{1}, \ldots, x_{n-q}\right)+Q_{q}+\mathrm{B}_{q}\left(x_{n-q+1}, \ldots, x_{n}\right),
$$

the first and last summands are already known to be subalgebras with mutually vanishing Lie brackets (i.e., the Lie brackets between them are 0). Furthermore, $Q_{q}$ is normalized by the first summand, and $\left[Q_{q}, \mathrm{~B}_{q}\left(x_{n-q+1}, \ldots\right.\right.$, 
$\left.\left.x_{n}\right)\right] \subset \mathrm{B}_{0}\left(x_{1}, \ldots, x_{n-q}\right)+Q_{q}$. So we only need to prove that $Q_{q}$ is a subalgebra. For this, it is sufficient to check that the operator $\sharp$ is closed on $\bar{\Delta}\{Q\}_{+}$. By a straightforward and easy computation, one sees that $\sharp$ is closed on the latter.

\subsection{Standard Borel subalgebras}

In this subsection, we prove that all $\mathrm{B}_{q}$ are Borel subalgebras of $W(n)$.

Proposition 3.3. Maintain the notations as before. All Borel subspaces $B_{r}$, $r=0,1,2, \ldots, n$, are Borel subalgebras of $W(n)$, called the standard Borel subalgebras.

Proof. By the definition of Borel subspaces, every Borel subspace contains the maximal torus $\mathfrak{t}_{0}$ of $W(n)$. According to Lemma 3.2(4), the Borel subspaces $\mathrm{B}_{q}$ are subalgebras of $W(n)$. It remains to show that any Borel subspace $\mathrm{B}_{q}$ is solvable and maximal among the solvable subalgebras of $W(n)$.

(1) We want to prove that all the subalgebras $B_{q}$ are solvable. By Lemma 2.4. we know that $\mathrm{B}_{0}$ is a solvable subalgebra. As for $\mathrm{B}_{n}$, we first observe that $\mathrm{B}_{n}^{(n+1)} \subset \mathrm{B}_{0}$, from which it follows that $\mathrm{B}_{n}^{(n+1)}$ is solvable. Hence $\mathrm{B}_{n}$ itself is solvable.

For an arbitrary $q$, recall $\mathrm{B}_{q}=\mathrm{B}_{0}\left(x_{1}, \ldots, x_{n-q}\right)+Q_{q}+\mathrm{B}_{q}\left(x_{n-q+1}, \ldots, x_{n}\right)$. The first and last summands have been known to be solvable subalgebras. Note that the middle summand subalgebra $Q_{q}$ is normalized by the first one and that the sum of the first and second summands are normalized by the third one. So we only need to verify that $Q_{q}$ is solvable. This follows from the inclusion $Q_{q}^{(q)} \subset W(n)_{1}$ (note that $W(n)_{1}$ is solvable).

(2) We want to prove that all solvable subalgebras $\mathrm{B}_{q}$ are maximal.

For $\mathrm{B}_{0}=\mathfrak{b}+W(n)_{1}$, the maximality is easily seen. Actually, if a solvable subalgebra $\mathfrak{H}$ contains $B_{0}$, then $\mathfrak{H}$ is $\mathfrak{t}_{0}$-graded by the homogenous condition. Then $\mathfrak{H}=\sum_{q} \mathfrak{H}_{[q]}$ with $\mathfrak{H}_{[q]}=\mathfrak{H} \cap W(n)_{[q]}$. The assumption of $\mathfrak{H}$ being solvable implies that $\mathfrak{H}_{[-1]}$ must be zero. Otherwise $\mathfrak{H}$ must contain a certain $\partial_{i}$, and thereby the subalgebra generated by $\partial_{i}, x_{i} \partial_{i}, x_{i}^{2} \partial_{i}$, which is not solvable. Hence $\mathfrak{H} \subset W(n)_{0}$. On the other hand, $\mathfrak{H}_{[0]}$ is solvable in $W(n)_{[0]}$. However $\mathfrak{b} \subset \mathfrak{H}_{[0]} \subset W(n)_{[0]}$, and $\mathfrak{b}$ is already a Borel subalgebra of $W(n)_{[0]}$. Hence $\mathfrak{H}_{[0]}$ coincides with $\mathfrak{b}$. Thus $\mathfrak{H}=\mathrm{B}_{0}$. 
As for $\mathrm{B}_{n}$, recall

$$
\mathrm{B}_{n}=W(n)_{[-1]}+\mathfrak{b}+\sum_{q=1}^{n} \sum_{\mathbf{a}(q)} \mathbb{K} \mathbf{x}^{\mathbf{a}(q)} \partial_{q}
$$

with the notation $\mathbf{x}=\left(x_{1}, \ldots, x_{n}\right), \mathbf{a}(q)=\left(a_{1}, \ldots, a_{q}, 0, \ldots, 0\right) \in \mathbb{P}^{n}$ with $|\mathbf{a}(q)|>1, a_{i}$ runs through $\mathbb{P}$ for $i=1, \ldots, q-1$, and $a_{q}=0,1$. Suppose there is a solvable algebra $\mathfrak{H} \supseteq \mathrm{B}_{n}$. Similar to the above argument, we have that $\mathfrak{H}=\sum_{q} \mathfrak{H}_{[q]}$ such that $\mathfrak{H}_{[q]}=\mathfrak{H} \cap W(n)_{[q]} \supset\left(\mathrm{B}_{n}\right)_{[q]}$ with $\mathfrak{H}_{[-1]}=W(n)_{[-1]}$ and $\mathfrak{H}_{[0]}=\mathfrak{b}$. If $\mathfrak{H}$ contains $B_{n}$ properly, then there must be a non-zero element $X \in \mathfrak{H}_{\alpha} \subset \mathfrak{H}_{[s]}$ for $s>0$, which is not in $\left(\mathrm{B}_{n}\right)_{[s]}$. Therefore we can write

$$
X=\sum_{q=1}^{n} \sum_{\mathbf{b}(q)} C_{\mathbf{b}(q)} \mathbf{x}^{\mathbf{b}(q)} \partial_{q}
$$

where $C_{\mathbf{b}(q)} \in \mathbb{K}, \mathbf{b}(q)=\left(b_{1}, \ldots, b_{n}\right) \in \mathbb{P}^{n}$ satisfying either $\left(b_{q+1}, \ldots, b_{n}\right) \neq$ 0 , or $\left(b_{q+1}, \ldots, b_{n}\right)=0$ with $b_{q}>1$, as long as $C_{\mathbf{b}(q)} \neq 0$. Say, either $b_{t} \neq 0$ for some $t>q$, or $b_{q}=d \geq 2$. We will deduce a contradiction. By applying a suitable series of adjoint actions $\operatorname{ad} D$ on $X$ for some $D \in$ $\mathfrak{H}_{[-1]}=W_{[-1]}$, one of the following situations happens: either $\mathfrak{H}$ contains a subalgebra generated by $x_{t} \partial_{q}, x_{q} \partial_{t}, x_{t} \partial_{t}-x_{q} \partial_{q}$, or $\mathfrak{H}$ contains a subalgebra generated by $x_{q}^{2} \partial_{q}, x_{q} \partial_{q}, \partial_{q}$. But neither of these two subalgebras is solvable, which contradicts the solvability of $\mathfrak{H}$. Hence, $\mathfrak{H}$ must coincide with $\mathrm{B}_{n}$. This completes the proof of the maximality of $\mathrm{B}_{n}$.

Let us finally investigate $\mathrm{B}_{q}(0<q<n)$. We need to prove that for any solvable subalgebra $\mathfrak{H}$, the inclusion $\mathfrak{H} \supseteq \mathrm{B}_{q}$ implies the equality $\mathfrak{H}=\mathrm{B}_{q}$. Observe that $\mathfrak{H} \cap W\left(x_{1}, \ldots, x_{n-q}\right) \supseteq \mathrm{B}_{0}\left(x_{1}, \ldots, x_{n-q}\right)$. Along with a result just proved that $\mathrm{B}_{0}\left(x_{1}, \ldots, x_{n-q}\right)$ is a Borel subalgebra of $W\left(x_{1}, \ldots, x_{n-q}\right)$, it follows that $\mathfrak{H} \cap W\left(x_{1}, \ldots, x_{n-q}\right)=\mathrm{B}_{0}\left(x_{1}, \ldots, x_{n-q}\right)$. For the same reason, we have

$$
\mathfrak{H} \cap W\left(x_{n-q+1}, \ldots, x_{n}\right)=\mathrm{B}_{q}\left(x_{n-q+1}, \ldots, x_{n}\right) .
$$

Thus we have

$$
\mathrm{B}_{q} \subseteq \mathfrak{H} \subseteq \mathrm{B}_{0}\left(x_{1}, \ldots, x_{n-q}\right)+\overline{Q_{q}}+\mathrm{B}_{q}\left(x_{n-q+1}, \ldots, x_{n}\right)
$$


where $\overline{Q_{q}}=\sum_{i=1}^{n-q} \sum_{\mathbf{a}(i), \mathbf{b}(i)} \mathbb{K} \mathbf{u}^{\mathbf{a}(i)} \mathbf{w}^{\mathbf{b}(i)} \partial_{i}+\sum_{j=n-q+1}^{n} \sum_{\mathbf{a}(j) ; \mathbf{b}(j)} \mathbb{K} \mathbf{u}^{\mathbf{a}(j)} \mathbf{w}^{\mathbf{b}(j)} \partial_{j}$, $\mathbf{u}=\left(x_{1}, \ldots, x_{n-q}\right)$ and $\mathbf{w}=\left(x_{n-q+1}, \ldots, x_{n}\right)$, and $\mathbf{a}(-):=\left(a_{1}, \ldots, a_{n-q}\right)$ runs through $\mathbb{P}^{n-q}$ for $(-)=(i),(j)$, and $\mathbf{b}(-):=\left(b_{n-q+1}, \ldots, b_{n}\right)$ runs through $\mathbb{P}^{q}$ for $(-)=(i),(j)$, subject to the condition that $|\mathbf{a}(j)|>0$ and $|\mathbf{b}(i)|>0$.

Suppose $\mathfrak{H} \supsetneqq \mathrm{B}_{q}$. Then there must be some element $X \in \overline{Q_{q}} \backslash Q_{q}$. Comparing $\overline{Q_{q}}$ and $Q_{q}$, we have

$$
X=\sum_{i=1}^{n-q} \sum_{\mathbf{a}(i), \mathbf{b}(i)} C_{\mathbf{a}(i), \mathbf{b}(i)} \mathbf{u}^{\mathbf{a}(i)} \mathbf{w}^{\mathbf{b}(i)} \partial_{i},
$$

with $C_{\mathbf{a}(i), \mathbf{b}(i)} \in \mathbb{K}$, and with $\mathbf{a}(i), \mathbf{b}(i)$ violating the condition listed below (3.1) as long as $C_{\mathbf{a}(i), \mathbf{b}(i)} \neq 0$. This is to say, there exists $j: 1 \leqslant i<j \leqslant n-q$ satisfying $|\mathbf{a}(i)|=1=a_{j}$ while $C_{\mathbf{a}(i), \mathbf{b}(i)} \neq 0$. By the same arguments as before, we conclude that $\mathfrak{H}$ contains $x_{j} \partial_{i}, x_{i} \partial_{j}$ and $x_{i} \partial_{i}-x_{j} \partial_{j}$, and therefore it contains the subalgebra generated by these elements, which is not solvable. This contradicts the hypothesis that $\mathfrak{H}$ is solvable. Consequently, we must have $\mathfrak{H}=\mathrm{B}_{q}$. Thus, this completes the proof of the maximality of the solvable subalgebras $\mathrm{B}_{q}$.

\section{Conjugacy Classes of Homogeneous Borel Subalgebras}

Maintain the notations and conventions as before. Especially, the characteristic $p$ of the ground field $\mathbb{K}$ is assumed to be greater than 3 throughout this section.

4.1. In this section, we will prove that the standard Borel subalgebras are representatives of the conjugacy classes of all homogeneous Borel subalgebras. Let $\mathcal{B}$ be any given Borel subalgebra of $W(n)$. By the definition of Borel subalgebras, $\mathcal{B}$ contains a maximal torus. In view of Theorem 2.2, we introduce an invariant $r(\mathcal{B})$ as follows

$$
\mathrm{r}(\mathcal{B}):=\max \left\{r \mid \text { there exists } \sigma \in \operatorname{Aut}(W(n)) \text { suth that } \sigma\left(\mathfrak{t}_{r}\right) \subset \mathcal{B}\right\}
$$

for the $G$-conjugacy class of $\mathcal{B}$. The following conclusion is obvious. 
Lemma 4.1. $r\left(B_{r}\right)=r$.

4.2. Let us begin with two special cases $r(\mathcal{B})=0$ and $r(\mathcal{B})=n$.

Lemma 4.2. Assume $\mathcal{B}$ is a homogeneous Borel subalgebra of $W(n)$ with $r(\mathcal{B})=0$. Then $\mathcal{B} \cong B_{0}$.

Proof. Up to conjugation, we may assume $\mathcal{B} \supset \mathfrak{t}_{0}$. Since $\mathcal{B}$ is homogeneous, it admits a standard-graded structure. So $\mathcal{B}=\sum_{i} \mathcal{B}_{[i]}$. We claim that $\operatorname{dim} \mathcal{B}_{[-1]}=0$. Indeed, if there is a non-zero $D \in \mathcal{B}_{[-1]}$, then $D=\sum_{i} c_{t_{i}} \partial_{t_{i}}$ with $c_{t_{i}} \in \mathbb{K} \backslash\{0\}$ for $t_{i} \in\{1, \ldots, n\}$. These elements $\partial_{t_{i}}$ belong to $\mathcal{B}$ because $\left[x_{t_{i}} \partial_{t_{i}}, D\right]=-c_{t_{i}} \partial_{t_{i}} \in \mathcal{B}$. Hence $\left(1+x_{t_{i}}\right) \partial_{t_{i}} \in \mathcal{B}$, which contradicts the assumption $r(\mathcal{B})=0$. Therefore, $\mathcal{B}$ is contained in $W(n)_{0}$. The conclusion follows then from Lemma 2.4.

4.3. Let $\mathcal{B}$ be a homogeneous Borel subalgebra of $W(n)$ with $r(\mathcal{B})=n$. This is to say, $\mathcal{B} \supset \sigma\left(\mathfrak{t}_{n}\right)$ for some $\sigma \in \operatorname{Aut}(W(n))$ and $\mathfrak{t}_{n}=\mathbb{K}\left(1+x_{1}\right) \partial_{1}+\cdots+$ $\mathbb{K}\left(1+x_{n}\right) \partial_{n}$.

Lemma 4.3. $\mathcal{B}$ is conjugate to $B_{n}$.

Proof. We may assume $\mathcal{B} \supset \mathfrak{t}_{n}$ without loss of generality. Since $\mathcal{B}$ is homogeneous, it is $\mathfrak{t}_{n}$-graded. We will prove the lemma by induction on $k^{\prime}:=n-\operatorname{dim} \mathcal{B}_{[-1]}^{\left(\mathfrak{t}_{n}\right)}$ (recall the notation in $\left.\S 2.4\right)$. We additionally set $k=$ $\operatorname{dim} \mathcal{B}_{[-1]}^{\left(\mathfrak{t}_{n}\right)}$.

When $k^{\prime}=0$, i.e., $\operatorname{dim} \mathcal{B}_{[-1]}^{\left(\mathfrak{t}_{n}\right)}=n$, we have $\mathcal{B} \supset \mathfrak{t}_{0}$. Since $\mathcal{B}$ is homogeneous, it admits a standard-graded structure, i.e., $\mathcal{B}=\sum_{i} \mathcal{B}_{[i]}$. Furthermore, $\mathcal{B}_{[-1]}=W(n)_{[-1]}$ and $\mathcal{B}_{[0]} \supset \mathfrak{t}_{0}$. Set $\mathcal{B}_{0}=\sum_{i \geq 0} \mathcal{B}_{[i]}$. We claim that

(*) Under the conjugation arising from some permutation $\pi$ of $(12 \cdots n)$ (see Theorem 2.1(1)),

$$
\mathcal{B}_{0} \subset \sum_{i} \sum_{\mathbf{a}(i)} \mathbb{K} \mathbf{x}^{\mathbf{a}(i)} \partial_{\pi(i)}
$$

where $\mathbf{x}=\left(x_{\pi(1)}, \ldots, x_{\pi(n)}\right)$, and $\mathbf{a}(i)=\left(a_{1}, \ldots, a_{i}, 0, \ldots, 0\right) \in \mathbb{P}^{n}$ subject to the condition that $a_{i}=0$ or $a_{i}=1$. 
Indeed, if the claim $(*)$ is not true, since $\mathcal{B}$ is $\mathfrak{t}_{0}$-graded, we readily conclude by suitable ad $\partial_{s}$-actions $(s \in\{1, \ldots, n\})$ that one of the following cases occurs:

$(\dagger)$ there exist in $\mathcal{B}$ a pair of elements $x_{i} \partial_{j}$ and $x_{j} \partial_{i}$ with $i \neq j$.

$(\ddagger)$ there exists $x_{i}^{2} \partial_{i}$ in $\mathcal{B}$ for some $i$.

The former means that there is a triple $x_{i} \partial_{j}, x_{j} \partial_{i}$ and $x_{i} \partial_{i}-x_{j} \partial_{j}$ in $\mathcal{B}$; the latter means that there is a triple $\partial_{i}, x_{i} \partial_{i}$ and $x_{i}^{2} \partial_{i}$ in $\mathcal{B}$. Either situation contradicts the solvability of $\mathcal{B}$. Hence the claim $(*)$ is true. Thus, by a suitable permutation $\tau$ of $\left(x_{1}, \ldots, x_{n}\right)$, which gives rise to an automorphism of $W(n), \mathcal{B}$ is isomorphic to a solvable subalgebra of $\mathrm{B}_{n}$. Hence $\mathcal{B} \cong \mathrm{B}_{n}$ by the maximal solvability of Borel subalgebras. This completes the proof for $k^{\prime}=0$.

Now suppose $k^{\prime}>0$ (this is to say, $k<n$ ), and suppose that the statement is true for integers less than $k^{\prime}$ (or equivalently, for integers greater than $k$ ). Without loss of generality, we assume $\mathcal{B}_{[-1]}^{\left(\mathfrak{t}_{n}\right)}=\mathbb{K} \partial_{1}+\cdots+\mathbb{K} \partial_{k}$ (we may apply some $g \in \mathrm{GL}(n)$ to get this). Then $\mathcal{B} \supset \mathfrak{t}_{n-k}=\mathbb{K} x_{1} \partial_{1}+\cdots+$ $\mathbb{K} x_{k} \partial_{k}+\mathbb{K}\left(1+x_{k+1}\right) \partial_{k+1}+\cdots+\mathbb{K}\left(1+x_{n}\right) \partial_{n}$. Set $\mathcal{B}_{1}:=\mathcal{B} \cap W\left(x_{1}, \ldots, x_{k}\right)$ and $\mathcal{B}_{2}:=\mathcal{B} \cap W\left(x_{k+1}, \ldots, x_{n}\right)$. Then

$$
\begin{aligned}
& \mathcal{B}_{1} \supset \sum_{i=1}^{k} \mathbb{K} \partial_{i}+\sum_{i=1}^{k} \mathbb{K} x_{i} \partial_{i} ; \\
& W\left(x_{k+1}, \ldots, x_{n}\right)_{0}^{\left(\mathfrak{t}_{n-k}\right)} \supset \mathcal{B}_{2} \supset \sum_{i=k+1}^{n} \mathbb{K}\left(1+x_{i}\right) \partial_{i} .
\end{aligned}
$$

The remaining argument will be divided into two cases: whether or not $\mathcal{B}_{2}$ contains nilpotent elements.

The first case: There is no nilpotent element in $\mathcal{B}_{2}$. Then $\mathcal{B}_{2}=\mathbb{K}(1+$ $\left.x_{k+1}\right) \partial_{k+1}+\cdots+\mathbb{K}\left(1+x_{n}\right) \partial_{n}$. We write

$$
\mathcal{B}=\mathcal{B}_{1}+\sum_{\left(b_{1}, \ldots, b_{k} ; c_{k+1}, \ldots, c_{n} ; i\right) \in I} \mathbb{K} x_{1}^{b_{1}} \cdots x_{k}^{b_{k}}\left(1+x_{k+1}\right)^{c_{k+1}} \cdots\left(1+x_{n}\right)^{c_{n}} \partial_{i}+\mathcal{B}_{2}
$$


for a subset $I$ of $\Gamma=\mathbb{P}^{k} \times \mathbb{P}^{n-k} \times\{1, \ldots, k\}$, where $\mathbb{P}:=\{0,1, \ldots, p-1\}$. Denote the second summand on the right hand side of (4.1) by $\mathcal{B}_{1}^{\prime}$. Then surely $\mathcal{B}_{1}^{\prime} \subset \mathcal{B}$.

Let us introduce a new subset $\bar{I}$ of $\Gamma$ associated with $I$

$$
\begin{aligned}
& \bar{I}:=\left\{\left(b_{1}, \ldots, b_{k} ; \gamma_{k+1}, \ldots, \gamma_{n} ; i\right) \mid \gamma_{i}=0,1, \ldots, p-1 ; \forall i=k+1, \ldots, n\right. \\
&\text { if exists } \left.\left(b_{1}, \ldots, b_{k} ; c_{k+1}, \ldots, c_{n} ; i\right) \in I\right\} .
\end{aligned}
$$

In the following, we simply write elements of $\bar{I}$ as $\left(b_{1}, \ldots, b_{k} ; \infty, \ldots, \infty ; i\right)$ (this means that some $\left(b_{1}, \ldots, b_{k} ;-, \ldots,-; i\right)$ appears in $\left.I\right)$ where $\infty$ just stands for any number from $\mathbb{P}$. Note that the same notation $\infty$ in different entries does not mean the same number. Set

$$
\overline{\mathcal{B}_{1}^{\prime}}=\sum_{\left(b_{1}, \ldots, b_{k} ; \infty, \ldots, \infty ; i\right) \in \bar{I}} \mathbb{K} x_{1}^{b_{1}} \cdots x_{k}^{b_{k}}\left(1+x_{k+1}\right)^{\infty} \cdots\left(1+x_{n}\right)^{\infty} \partial_{i} .
$$

And set

$$
\overline{\mathcal{B}}=\mathcal{B}_{1}+\overline{\mathcal{B}_{1}^{\prime}}+\mathcal{B}_{2}
$$

We claim that

$$
\overline{\mathcal{B}} \text { is a solvable subalgebra of } W(n) \text { containing } \mathcal{B},
$$

therefore $\mathcal{B}$ coincides with $\overline{\mathcal{B}}$ by the maximality of $\mathcal{B}$. To prove the above claim, we first need to check that the linear subspace $\overline{\mathcal{B}}$ is a subalgebra. For this, note that $\mathcal{B}_{1}+\overline{\mathcal{B}_{1}^{\prime}}$ is normalized by $\mathcal{B}_{2}$. We only need to check that $\mathcal{B}_{1}+\overline{\mathcal{B}_{1}^{\prime}}\left(=\overline{\mathcal{B}_{1}^{\prime}}\right)$ is a subalgebra. In order to check this, we take any two elements $\bar{X}, \bar{X}^{\prime} \in \overline{\mathcal{B}_{1}^{\prime}}$ with $\bar{X}=x_{1}^{b_{1}} \cdots x_{k}^{b_{k}}\left(1+x_{k+1}\right)^{\infty} \cdots\left(1+x_{n}\right)^{\infty} \partial_{i}$, $\bar{X}^{\prime}=x_{1}^{b_{1}^{\prime}} \cdots x_{k}^{b_{k}^{\prime}}\left(1+x_{k+1}\right)^{\infty} \cdots\left(1+x_{n}\right)^{\infty} \partial_{j}$. Correspondingly, we can find two elements $X$ and $X^{\prime}$ in $\mathcal{B}_{1}^{\prime}$ defined as follows:

$$
\begin{aligned}
X & =x_{1}^{b_{1}} \cdots x_{k}^{b_{k}}\left(1+x_{k+1}\right)^{c_{k+1}} \cdots\left(1+x_{n}\right)^{c_{n}} \partial_{i} \\
X^{\prime} & =x_{1}^{b_{1}^{\prime}} \cdots x_{k}^{b_{k}^{\prime}}\left(1+x_{k+1}\right)^{c_{k+1}^{\prime}} \cdots\left(1+x_{n}\right)^{c_{n}^{\prime}} \partial_{j}
\end{aligned}
$$

for some $\left(c_{k+1}, \ldots, c_{n}\right),\left(c_{k+1}^{\prime}, \ldots, c_{n}^{\prime}\right) \in \mathbb{P}^{n-k}$. Then

$$
\left[\bar{X}, \bar{X}^{\prime}\right]=\left(1+x_{k+1}\right)^{\infty} \cdots\left(1+x_{n}\right)^{\infty}\left[x_{1}^{b_{1}} \cdots x_{k}^{b_{k}} \partial_{i}, x_{1}^{b_{1}^{\prime}} \cdots x_{k}^{b_{k}^{\prime}} \partial_{j}\right] \in \overline{\mathcal{B}}_{1}^{\prime}
$$


because $\left[X, X^{\prime}\right] \in\left[\mathcal{B}_{1}^{\prime}, \mathcal{B}_{1}^{\prime}\right] \subset \mathcal{B}_{1}^{\prime}$, and $\mathcal{B}^{\prime} \subset \mathcal{B}$ is $\mathfrak{t}_{n-k}$-graded. The solvability of $\overline{\mathcal{B}}$ follows from (4.2), along with the relations

$$
\left[\overline{\mathcal{B}}_{1}^{\prime}, \overline{\mathcal{B}}_{1}^{\prime}\right] \subset\left\{\sum\left(1+x_{k+1}\right)^{\infty} \cdots\left(1+x_{n}\right)^{\infty} X \mid X \in\left[\mathcal{B}_{1}^{\prime}, \mathcal{B}_{1}^{\prime}\right]\right\}
$$

and inductively for $i=2,3, \ldots$

$$
\overline{\mathcal{B}}_{1}^{\prime(i)} \subset\left\{\sum\left(1+x_{k+1}\right)^{\infty} \cdots\left(1+x_{n}\right)^{\infty} X \mid X \in \mathcal{B}_{1}^{(i)}\right\},
$$

and the solvability of $\mathcal{B}$. Hence, $\mathcal{B}=\overline{\mathcal{B}}$. On the other hand, the structure of $\overline{\mathcal{B}}$ implies that $\mathcal{B}+\mathbb{K} \partial_{n}=\overline{\mathcal{B}}+\mathbb{K} \partial_{n}$ is a solvable subalgebra of $W(n)$, which contradicts the maximal solvability of $\mathcal{B}$ because $\mathcal{B}+\mathbb{K} \partial_{n} \supsetneqq \mathcal{B}$ (note that $k<n)$. So the first case could not happen.

The second case: There exist some nilpotent elements in $\mathcal{B}_{2}$. In this case, $\mathcal{B}_{2} \supsetneqq \mathfrak{t}_{n-k}\left(x_{k+1}, \ldots, x_{n}\right)=\mathbb{K}\left(1+x_{k+1}\right) \partial_{k+1}+\cdots+\mathbb{K}\left(1+x_{n}\right) \partial_{n}$. Note that $\mathcal{B}$ is a maximal solvable subalgebra containing $\mathfrak{t}_{n}$, and $\mathcal{B}_{2}$ is $\mathfrak{t}_{n-k}\left(x_{k+1}, \ldots, x_{n}\right)$ graded. We claim that there exists in $\mathcal{B}_{2}$ a nilpotent element of "monomial form"

$$
X=\left(1+x_{k+1}\right)^{a_{k+1}} \cdots\left(1+x_{n}\right)^{a_{n}} \partial_{q},
$$

which satisfies $a_{q} \neq 1$ (subsequently called the $q$-condition). To verify this, we first have, by a direct computation, that any derivation of the form $\left(1+x_{q}\right)\left(\prod_{i \neq q}\left(1+x_{i}\right)^{a_{i}}\right) \partial_{q}$ is non-nilpotent, thereby a nilpotent element of "monomial form" must satisfy the q-condition. Next, we show that such a nilpotent element of "monomial form" indeed exists in $\mathcal{B}_{2}$. By employing the $\mathfrak{t}_{n-k}\left(x_{k+1}, \ldots, x_{n}\right)$-grading, we can first take a nilpotent element $X^{\prime}$ to be a linear combination of some "monomial form" elements of a $\mathfrak{t}_{n-k}\left(x_{k+1}, \ldots, x_{n}\right)$-grading $\left(d_{k+1}, \ldots, d_{n}\right)$. Under a suitable conjugation similar to the forthcoming (4.4) (only involving $x_{k+1}, \ldots, x_{n}$ and satisfying the forthcoming $(\Phi-2)$ ), we can get a "monomial" summand from the combination such that its $\mathfrak{t}_{n-k}\left(x_{k+1}, \ldots, x_{n}\right)$-grading is different from the other summands. Then such a "monomial" summand must be a nilpotent element in $\mathcal{B}_{2}$. We take it as the desired $X$ (the above computation is trivial and tedious, we omit the details here).

With such an $X \in \mathcal{B}_{2}$ as in (4.3), we can further see that $\mathcal{B}$ is conjugate to some $\mathcal{B}^{\prime}$ with $\operatorname{dim}\left(\mathcal{B}^{\prime}\right)_{[-1]}^{\left(t_{n}\right)}=k+1$ as follows. 
By the same way as in $\S 2.2$, we define an automorphism $\varphi$ of the truncated polynomial algebra $A(n)$ via

$$
\begin{aligned}
& \varphi: x_{j} \mapsto x_{j} \text { for } j \in\{1, \ldots, n\} \backslash\{q\}, \\
& \varphi: x_{q} \mapsto\left(1+x_{q}\right) \prod_{j \in\{k+1, \ldots, n\}, j \neq q}\left(1+x_{j}\right)^{d_{j}}-1,
\end{aligned}
$$

where $d_{j}=d_{q}\left(p-a_{j}\right) \in\{0,1, \ldots, p-1\}$ with $d_{q}\left(a_{q}-1\right) \equiv 1 \bmod p$. Then $\varphi$ induces an automorphism $\bar{\varphi}$ of $W(n)$, denoted by $\Phi$. Then $\left.\Phi\right|_{\mathcal{B}_{1}}$ is the identity map. We have

$$
\begin{aligned}
\Phi\left(\partial_{q}\right) & =\varphi \circ \partial_{q} \circ \varphi^{-1} \\
& =\prod_{j \in\{k+1, \ldots, n\}, j \neq q}\left(1+x_{j}\right)^{p-d_{j}} \partial_{q} .
\end{aligned}
$$

And then we have

$$
\begin{aligned}
\Phi(X) & =\left(\prod_{j \in\{k+1, \ldots, n\}, j \neq q}\left(1+x_{j}\right)^{a_{j}}\right) \varphi\left(\left(1+x_{q}\right)^{a_{q}}\right) \Phi\left(\partial_{q}\right) \\
& =\left(1+x_{q}\right)^{a_{q}} \partial_{q} .
\end{aligned}
$$

Furthermore, $\Phi\left(\left(1+x_{j}\right) \partial_{j}=\left(1+x_{j}\right) \partial_{j}\right.$ for $j \in\{k+1, \ldots, n\} \backslash\{q\}$. And

$$
\Phi\left(\left(1+x_{q}\right) \partial_{q}\right)=\varphi\left(1+x_{q}\right) \Phi\left(\partial_{q}\right)=\left(1+x_{q}\right) \partial_{q}
$$

Hence $\Phi$ satisfies the following properties

$\left.(\Phi-1) \Phi\right|_{\mathfrak{t}_{n-k}\left(x_{k+1}, \ldots, x_{n}\right)}=$ identity.

$\left(\Phi\right.$-2) Under $\Phi, \Phi(\mathcal{B})=\mathcal{B}_{1}+\Phi\left(\mathcal{B}_{2}\right)$, which contains $\mathfrak{t}_{n}$, and intersects with $W(n)_{[-1]}^{(n)}$ at $\sum_{i=1}^{k} \mathbb{K} \partial_{i}$.

$(\Phi-3)$ It is a specially important consequence that $\Phi\left(\mathcal{B}_{2}\right)$ admits a nilpotent element $\Phi(X)=\left(1+x_{q}\right)^{a_{q}} \partial_{q}$.

Next, we define an automorphism $\Psi$ of $W(n)$ via

$$
\psi\left(x_{j}\right)= \begin{cases}x_{j}, & \text { if } j \neq q \\ \left(1+x_{q}\right)^{b_{q}}-1, & \text { if } j=q\end{cases}
$$


where $b_{q} \in\{1, \ldots, p-1\}$ with $\left(p-a_{q}+1\right) b_{q} \equiv 1 \bmod p$. By a straightforward computation as in (2.6), we have $\Psi \circ \Phi(\mathcal{B}) \supset \mathfrak{t}_{n}$ with $\operatorname{dim} \Psi \circ \Phi(\mathcal{B})_{[-1]}^{\left(\mathfrak{t}_{n}\right)}=k+1$. The induction hypothesis yields that $\Psi \circ \Phi(\mathcal{B})$ is conjugate to $\mathcal{B}_{n}$. This completes the proof.

4.4. Now we are in the position to investigate the general case.

Lemma 4.4. Let $\mathcal{B}$ be any given homogeneous Borel subalgebra of $W(n)$. Then $\mathcal{B}$ is conjugate to $B_{r}$, where $r=r(\mathcal{B})$.

Proof. If $r=n$, then the statement holds, thanks to Lemma 4.3. In the following, we assume $r<n$. Up to conjugation, we may assume that $\mathcal{B}$ contains $\mathfrak{t}_{r}=\mathbb{K} x_{1} \partial_{1}+\mathbb{K} x_{2} \partial_{2}+\cdots+\mathbb{K} x_{n-r} \partial_{n-r}+\mathbb{K}\left(1+x_{n-r+1}\right) \partial_{n-r+1}+$ $\cdots+\mathbb{K}\left(1+x_{n}\right) \partial_{n}$. Since $\mathcal{B}$ is homogeneous, we have that $\mathcal{B}$ is $\mathfrak{t}_{r}$-graded. According to the assumption, we have the following observation:

$(\mathrm{r}-0) \mathcal{B}_{[-1]}^{\left(\mathrm{t}_{r}\right)} \subset \mathbb{K} \partial_{n-r+1}+\cdots+\mathbb{K} \partial_{n}$.

Note that $\mathcal{B}_{[0]}^{\left(\mathfrak{t}_{r}\right)} \cap W\left(x_{1}, \ldots, x_{n-r}\right)$ is a solvable subalgebra of $W\left(x_{1}, \ldots, x_{n-r}\right)_{[0]}$ containing the maximal torus $\mathfrak{t}_{0}\left(x_{1}, \ldots, x_{n-r}\right)$. And $G_{0}=$ $\mathrm{GL}(n, \mathbb{K}) \supset \mathrm{GL}(n-r, \mathbb{K}) \times \mathrm{GL}(r)$. By a result on the conjugacy classes of Borel subalgebra of classical Lie algebras (cf. [8, §11.3-4]), without loss of generality, we can additionally make the following assumption:

$(\mathrm{r}-1) \mathcal{B}_{[0]}^{\left(\mathfrak{t}_{r}\right)} \cap W\left(x_{1}, \ldots, x_{n-r}\right) \subset \sum_{i \leqq j ; i, j=1, \ldots, n-r} \mathbb{K} x_{i} \partial_{j}$.

Set $\mathcal{B}_{1}=\mathcal{B} \cap W\left(x_{1}, \ldots, x_{n-r}\right)$. Then $\mathcal{B}_{1}$ is solvable, and $\mathfrak{t}_{0}\left(x_{1}, \ldots, x_{n-r}\right) \subset$ $\mathcal{B}_{1} \subset W(n-r)_{0}$. According to Lemma 2.4, without loss of generality, we can additionally make the following assumption:

$(\mathrm{r}-2) \mathcal{B}_{1} \subset \mathrm{B}_{0}\left(x_{1}, \ldots, x_{n-r}\right)$.

Set $\mathcal{B}_{2}=\mathcal{B} \cap W\left(x_{n-r+1}, \ldots, x_{n}\right)$. Then $\mathcal{B}_{2}$ is solvable and contains the maximal torus

$$
\mathfrak{t}_{r}\left(x_{n-r+1}, \ldots, x_{n}\right)
$$

of $W\left(x_{n-r+1}, \ldots, x_{n}\right)$. Observe that

$$
\operatorname{Aut}\left(W\left(x_{1}, \ldots, x_{n-r}\right)\right) \times \operatorname{Aut}\left(W\left(x_{n-r+1}, \ldots, x_{n}\right)\right) \subset \operatorname{Aut}(W(n)) .
$$


So by taking Lemma 4.3 into account, we may additionally make the following assumption:

$(\mathrm{r}-3) \mathcal{B}_{2} \subset \Theta\left(\mathrm{B}_{r}\left(x_{n-r+1}, \ldots, x_{n}\right)\right)$ for a certain $\Theta \in \operatorname{Aut}\left(W\left(x_{n-r+1}, \ldots, x_{n}\right)\right)$.

Set $\mathbf{x}=\left(x_{1}, \ldots, x_{n-r}\right)$ and $\mathbf{y}=\left(y_{n-r+1}, \ldots, y_{n}\right)$ for $y_{i}=1+x_{i}$. Consider the subspace $Q$ below (keeping the notations in Conventions 3.1):

$$
Q=\sum_{i=1}^{n-r} \sum_{\mathbf{a}(i), \mathbf{b}(i)} \mathbb{K} \mathbf{x}^{\mathbf{a}(i)} \mathbf{y}^{\mathbf{b}(i)} \partial_{i}+\sum_{j=n-r+1}^{n} \sum_{\mathbf{a}(j) ; \mathbf{b}(j)} \mathbb{K} \mathbf{x}^{\mathbf{a}(j)} \mathbf{y}^{\mathbf{b}(j)} \partial_{j},
$$

where $\mathbf{a}(i):=\left(a_{1}, \ldots, a_{n-r}\right) \in \mathbb{P}^{n-r}$ is subject to the condition that either $|\mathbf{a}(i)|>1$ or $|\mathbf{a}(i)|=1=a_{1}+\cdots+a_{i}$, while $\mathbf{a}(j) \in \mathbb{P}^{n-r}$ is subject to the condition $|\mathbf{a}(j)|>0$, and $\mathbf{b}(-):=\left(b_{n-r+1}, \ldots, b_{n}\right)$ runs through $\mathbb{P}^{r}$ for $(-)=(i),(j)$. It is readily seen that $Q$ is stable under the action of $\operatorname{Aut}\left(W\left(x_{n-r+1}, \ldots, x_{n}\right)\right)$, and that the following statements hold

(r-4) $Q$ is a subalgebra normalizing $\mathrm{B}_{0}\left(x_{1}, \ldots, x_{n-r}\right)$ and $Q+\mathrm{B}_{0}\left(x_{1}, \ldots, x_{n-r}\right)$ normalizes $\Theta\left(\mathrm{B}_{r}\left(x_{n-r+1}, \ldots, x_{n}\right)\right)$.

$(\mathrm{r}-5) \Theta\left(\mathrm{B}_{r}\right)=\mathrm{B}_{0}\left(x_{1}, \ldots, x_{n-r}\right)+Q+\Theta\left(\mathrm{B}_{r}\left(x_{n-r+1}, \ldots, x_{n}\right)\right)$ (note that $\Theta$ can be naturally regarded as an automorphism of $W(n))$.

Set $\mathcal{B}_{Q}:=\mathcal{B}_{1}+Q+\mathcal{B}_{2}$. Then $\mathcal{B}_{Q} \subset \Theta\left(\mathrm{B}_{r}\right)$. From (r-2), (r-3) and (r-4), we know that $\mathcal{B}_{Q}$ is a solvable subalgebra. We will finally show the following inclusion relation

$(\mathrm{r}-6) \mathcal{B}_{Q} \supset \mathcal{B}$.

In order to prove $(\mathrm{r}-6)$, we first claim that $\mathcal{B}$ may contain neither elements like $Y$ nor elements like $Z$, where $Y$ and $Z$ are presented as

$$
Y=\mathbf{y}^{\mathbf{b}(q)} \partial_{q} \text { with } q \in\{1,2, \ldots, n-r\}
$$

and

$$
Z=x_{m} \mathbf{y}^{\mathbf{b}(q)} \partial_{q} \text { with } m, q \in\{1,2, \ldots, n-r\}, m>q
$$

We verify the claim by deriving a contradiction. Suppose the claim is not true and the elements of the form (4.5) or (4.6) occur in $\mathcal{B}$. First, suppose that in $\mathcal{B}$ there exists an element $Y$ of the form (4.5). Clearly, $|\mathbf{b}(q)|>0$ 
by $(\mathrm{r}-0)$. For $Y=\left(1+x_{n-r+1}\right)^{b_{n-r+1}} \cdots\left(1+x_{n}\right)^{b_{n}} \partial_{q}$. According to the argument of $\S 2.2$, one can consider an automorphism $\Omega$ of $W(n)$ induced by $\omega \in \operatorname{Aut}(A(n))$, which is defined via

$$
\begin{aligned}
& \omega: x_{i} \mapsto x_{i} \text { for } i \in\{1, \ldots, n\} \backslash\{q\}, \\
& \omega: x_{q} \mapsto x_{q} \prod_{j \in\{n-r+1, \ldots, n\}}\left(1+x_{j}\right)^{b_{j}}
\end{aligned}
$$

Then $\Omega(Y)=\partial_{q}$, while $\Omega\left(x_{q} \partial_{q}\right)=x_{q} \partial_{q}$, and we have $\Omega(\mathcal{B}) \supset \sum_{i=1, i \neq q}^{n-r} \mathbb{K} x_{i} \partial_{i}$ $+\mathbb{K}\left(1+x_{q}\right) \partial_{q}+\sum_{i=n-r+1}^{n} \mathbb{K}\left(1+x_{i}\right) \partial_{i} \cong \mathfrak{t}_{r+1}$, which contradicts the assumption $r(\mathcal{B})=r$. Thus we have proved that $\mathcal{B}$ does not contain any elements of the form (4.5).

For elements of the form (4.6), we first conclude that

$\mathcal{B}$ does not contain any pair of elements of the forms:

$$
\begin{aligned}
& Z(m, q)=x_{m}\left(1+x_{n-r+1}\right)^{b_{n-r+1}} \cdots\left(1+x_{n}\right)^{b_{n}} \partial_{q} \\
& Z(q, m)=x_{q}\left(1+x_{n-r+1}\right)^{c_{n-r+1}} \cdots\left(1+x_{n}\right)^{c_{n}} \partial_{m}
\end{aligned}
$$

with $m, q \in\{1,2, \ldots, n-r\}, m>q$. Otherwise, suppose that $\mathcal{B}$ contains a pair of elements as above. Then one can easily find a non-solvable subalgebra in $\mathcal{B}$ arising from such pairs, which contradicts the solvability of $\mathcal{B}$. Combining the statement (4.7) with the assumption ( $\mathrm{r}-1)$, we finally conclude that the maximal solvable subalgebra $\mathcal{B}$ does not contain any elements of the form (4.6) (if necessary, we change the order of the indeterminants $x_{i}$ for $i=1, \ldots, n-r$, which gives rise to some automorphism of $W(n))$. This verifies the claim.

In order to complete the proof of $(\mathrm{r}-6)$, it remains to show that $\mathcal{B}$ does not contain any $\mathbb{K}$-linear combination of some elements of the form (4.5) or (4.6). Notice that all summands in such a combination admit different $\mathfrak{t}_{r}$-gradings (different elements of the form (4.5) or (4.6) admit different $\mathfrak{t}_{r}$ gradings). On the other hand, since $\mathcal{B}$ is homogeneous, it is $\mathfrak{t}_{r}$-graded. If $\mathcal{B}$ contains a combination of different $\mathfrak{t}_{r}$-graded summands, then $\mathcal{B}$ contains all its summands. This contradicts what we just concluded. So $\mathcal{B}$ does not contain such a combination. Thus, comparing $\mathcal{B}_{q}$ and $\mathcal{B}$, we have $\mathcal{B}_{Q} \supset \mathcal{B}$. We have finished the proof of (r-6). 
It follows from $(\mathrm{r}-2)-(\mathrm{r}-6)$ that $\mathcal{B} \subset \mathcal{B}_{Q} \subset \Theta\left(\mathrm{B}_{r}\right)$. The maximality of $\mathcal{B}$ implies $\mathcal{B}=\Theta\left(B_{r}\right)$. This completes the proof.

We have a direct consequence

Corollary 4.5. The following statements hold.

(1) Any homogeneous Borel subalgebra of $W(n)$ contains a maximal torus conjugate to $\mathfrak{t}_{0}$.

(Therefore, for a given homogeneous Borel subalgebra $\mathcal{B}$ of $W(n)$, we can take some $\mathcal{B}^{0}$ conjugate to $\mathcal{B}$ such that $\mathcal{B}^{0} \supset \mathfrak{t}_{0}$.)

(2) If we set $d(\mathcal{B})=\operatorname{dim} \mathcal{B}_{[-1]}^{0}$, then $d(\mathcal{B})=r(\mathcal{B})$.

So, there is a constant $\mathrm{d}(\mathcal{B})=\mathrm{r}(\mathcal{B})$ associated with the conjugacy class of a homogeneous Borel subalgebra $\mathcal{B}$.

4.5. By Lemma 4.4, Corollary 4.5 and [2, Proposition 3.3] (note that in [2], the order of the parameters in the subscripts of the notations $\left\{\mathfrak{t}_{r}\right\}$ is reversed to the one used in the present paper), we have

Theorem 4.6. Assume that $\mathfrak{g}=W(n), G=\operatorname{Aut}(W(n))$, and that the characteristic $p$ of the ground field $\mathbb{K}$ is greater than 3 . Then the following statements hold.

(1) All standard Borel subalgebras $B_{i}, i=0,1, \ldots, n$ are homogeneous.

(2) There are $(n+1)$ conjugacy classes of homogeneous Borel subalgebras of $W(n)$ under $G$. The standard Borel subalgebras $B_{i}, i=0,1, \ldots, n$ are representatives of the $(n+1)$ conjugacy classes.

(3) There is only one conjugacy class of generic homogeneous Borel subalgebras, which is conjugate to $B_{n}$.

Remark 4.7. In view of [7, Theorem D], it is reasonable to expect that all maximal solvable subalgebras of $W(n)$ are Borel subalgebras if and only if $p>n$. In the case $p \leqslant n$, the problem of determining the conjugacy classes of maximal solvable subalgebras seems to be much harder. 


\section{Solvable Subgroups Associated with Standard Borel Subalgebras}

Let $(\mathfrak{g},[p])$ be a restricted Lie algebra, and let $G$ the adjoint group of $\mathfrak{g}$. We are especially interested in $\operatorname{Stab}_{G}\left(\mathcal{B}_{\text {gen }}\right)$ and $G / \operatorname{Stab}_{G}\left(\mathcal{B}_{\text {gen }}\right)$ for a generic Borel subalgebra $\mathcal{B}_{\text {gen }}$ when $\mathfrak{g}$ admits generic tori, where $\operatorname{Stab}_{G}\left(\mathcal{B}_{\text {gen }}\right)$ denotes the subgroup of $G$ consisting of elements stabilizing $\mathcal{B}_{\text {gen }}$. In this section, we investigate the subgroups $\operatorname{Stab}_{G}\left(\mathcal{B}_{r}\right)$ associated with standard Borel algebras in the case $\mathfrak{g}=W(n)$. Keep the notation as in Theorem 2.1.

5.1. For the standard homogeneous Borel subalgebras $\mathrm{B}_{r}$ of $W(n)$, set $S_{r}=\operatorname{Stab}_{G}\left(\mathrm{~B}_{r}\right), r=0,1, \ldots, n$. Keeping in mind that $G_{0}$ is isomorphic to $\operatorname{GL}(n, \mathbb{K})$ (cf. Theorem 2.1), we have a standard Borel subgroup $B_{0}$ of $G_{0}$ which corresponds to the one consisting of invertible upper-triangular matrices of $\mathrm{GL}(n, \mathbb{K})$. Then we have the following general description of $S_{r}$.

Proposition 5.1. Let $B_{0}$ be the standard Borel subgroup of $G_{0}$ corresponding to the one consisting of invertible upper-triangular matrices of $G L(n, \mathbb{K})$. The following statements hold.

(1) The solvable subgroup $S_{0}$ coincides with the Borel subgroup $B_{0} \ltimes U$ of $G$.

(2) For $r=0,1, \ldots, n, S_{r}=B_{0} \ltimes U_{r}$ is a connected subgroup of the Borel subgroup $S_{0}$, where $U_{r}=U \cap \operatorname{Stab}_{G}\left(B_{r}\right)$.

Proof. The first assertion is immediate. For (2), it is easily verified that $S_{r}=B_{0} \ltimes U_{r}$. It remains to prove the connectedness of $U_{r}$. We identify $B_{0}$ with the standard Borel subgroup of $\operatorname{GL}(n, \mathbb{K})$ consisting of invertible upper-triangular matrices, which has a maximal torus group $T_{0}$ consisting of invertible diagonal matrices. Obviously, $T_{0}$ normalizes $U_{r}$. Take $\tau(t)=\operatorname{diag}(t, t, \ldots, t) \in T_{0}$ for a given $t \in \mathbb{K}^{\times}:=\mathbb{K} \backslash\{0\}$. Given a unipotent element $g \in U_{r}$, we can write $g=\mathrm{id}_{A(n)}+\phi$ with $\phi \in \operatorname{End}(A(n))$ satisfying $\phi\left(x_{i}\right) \in \sum_{j>1} A_{[j]}$ for $i=1, \cdots, n$, where $A(n)_{[j]}$ is the subspace spanned by homogeneous truncated polynomials of degree $j$ as defined in $\S 2.1$. Furthermore, we can write $\phi=\sum_{j>1} \phi_{j}$ with $\phi_{j}$ mapping $A_{[1]}$ to $A_{[j]}$. Then we get

$$
\tau(t) g \tau(t)^{-1}=\mathrm{id}_{A(n)}+\sum_{j>1} t^{j-1} \phi_{j}
$$


for all $t \in \mathbb{K}^{\times}$. We extend the map sending $t$ to $\tau(t) g \tau(t)^{-1}$ to a morphism $\mathbb{K} \rightarrow \operatorname{End}\left(A_{n}\right)$. Clearly, both $\operatorname{id}_{A(n)}$ and $g$ belong to the image. Thus $g$ belongs to the same connected component of $U_{r}$ as $\operatorname{id}_{A(n)}$. The arbitrariness of $g$ implies that $U_{r}$ is connected.

5.2. Set $\mathfrak{g}=W(n)$ and $G=\operatorname{Aut}(W(n))$ described as in Theorem 2.1, We use the notation $B_{\text {gen }}$ for $\operatorname{Stab}_{G}\left(\mathrm{~B}_{n}\right)$ instead of $S_{n}$.

Proposition 5.2. By identifying $G$ and the automorphism group of $A(n)$, $B_{\text {gen }}$ can be regarded as a subgroup of the isotropy group of the following flag of algebras

$$
\mathbb{K}\left[x_{1}, \ldots, x_{n}\right] \supset \mathbb{K}\left[x_{1}, \ldots, x_{n-1}\right] \supset \cdots \supset \mathbb{K}\left[x_{1}\right]
$$

Furthermore, $B_{\text {gen }}=B_{0} \ltimes U_{n}$, with

$$
U_{n}=\left\{g \in U \mid(g-i d)\left(x_{i}\right) \in\left(\mathbb{K}+\mathbb{K} x_{i}\right) \mathbb{K}\left[x_{1}, \ldots, x_{i-1}\right], i=1, \ldots, n\right\} .
$$

Proof. It is clear that $B_{\text {gen }}$ contains both $B_{0}$ and $U_{n}$. Hence it contains also $B \ltimes U_{n}$. Next we prove the inverse inclusion.

For any $\sigma \in B_{\text {gen }}$, we can write $\sigma=\sigma_{0} \circ \sigma_{1}$ with $\sigma_{0} \in G_{0}$ and $\sigma_{1} \in U$ by Theorem 2.1. So $\sigma_{0}$ stabilizes $\mathfrak{b} \subset \mathrm{B}_{n}$, which means that $\sigma_{0} \in B_{0} \subset$ $B_{\text {gen }}$. Thus $\sigma_{1} \in B_{\text {gen. }}$. It remains to show that $\sigma_{1} \in U_{n}$. Suppose $\sigma_{1}$ is not in $U_{n}$. Then there exists some $i$ such that $\sigma_{1}\left(x_{i}\right)=x_{i}+x_{j} f_{1}+$ $x_{i}^{2} f_{2}+f_{3}$ with $j>i, f_{1} \in \mathbb{K}\left[x_{1}, \ldots, x_{n}\right], f_{2} \in \mathbb{K}\left[x_{1}, \ldots, \widehat{x_{j}}, \ldots, x_{n}\right], f_{3} \in$ $\left(\mathbb{K}+\mathbb{K} x_{i}\right) \mathbb{K}\left[x_{1}, \ldots, \widehat{x_{i}}, \ldots, \widehat{x_{j}}, \ldots, x_{n}\right]$, and $f_{1}, f_{2}$ are not all zero. Here the notations $\widehat{x_{i}}, \widehat{x_{j}}$ mean omitting the indeterminants $x_{i}, x_{j}$ respectively. Note that $\sigma_{1} \in U$. By a straightforward computation, $\sigma_{1}^{-1}\left(x_{i}\right)=x_{i}+x_{j} g_{1}+x_{i}^{2} g_{2}+$ $g_{3}$, where $g_{1}, g_{2}, g_{3} \in A(n)$ are subject to the same conditions as the ones for $f_{1}, f_{2}$ and $f_{3}$. Then $\bar{\sigma}_{1}\left(x_{i} \partial_{i}\right)=x_{i} \partial_{i}+\left(x_{j} f_{1}+x_{i}^{2} f_{2}+f_{3}\right) \partial_{i}+*$ does not fall in $\mathrm{B}_{n}$. This is a contradiction. Hence $\sigma$ lies in $B_{0} \ltimes U_{n}$. This completes the proof.

\section{Additional Notation List}

For the truncated polynomial algebra $A(n)$ and its derivation algebra $W(n)$. 
- $\mathbb{P}=\{0,1 \ldots, p-1\}$.

- $\epsilon_{i}=\left(\delta_{i, 1}, \ldots, \delta_{i, n}\right) \in \mathbb{P}^{n}, \delta_{i, j}=1$ if $i=j$, and 0 otherwise.

- $A(n)=\mathbb{K}\left[T_{1}, \ldots, T_{n}\right] /\left(T_{1}^{p}, \ldots, T_{n}^{p}\right)$.

- $x_{i}=T_{i}+\left(T_{1}^{p}, \ldots, T_{n}^{p}\right) \in A(n), y_{i}=x_{i}+1$, and $z_{i}=x_{i}$ or $y_{i}$ according to the prescribed, $i=1, \ldots, n$.

- $\bar{\varphi}$ is an automorphism in $W(n)$ induced from $\varphi \in \operatorname{Aut}(A(n))$.

- $\mathbf{x}^{\mathbf{a}}=x_{1}^{a_{1}} \cdots x_{n}^{a_{n}} ; \mathbf{y}^{\mathbf{a}}=y_{1}^{a_{1}} \cdots y_{n}^{a_{n}} ;$ and $\mathbf{z}^{\mathbf{a}}=z_{1}^{a_{1}} \cdots z_{n}^{a_{n}}$ all in $A(n)$ for $\mathbf{a}=\left(a_{1}, \ldots, a_{n}\right) \in \mathbb{P}^{n}$.

For maximal tori and related gradations:

- $\mathfrak{t}_{r}=\sum_{i=1}^{n-r} \mathbb{K} x_{i} \partial_{i}+\sum_{i=(n-r)+1}^{n} \mathbb{K}\left(1+x_{i}\right) \partial_{i}, r=0,1, \ldots, n$ (see $\left.\S 2.3\right)$.

- $\mathfrak{H}_{[i]}^{\left(\mathfrak{t}_{r}\right)}: \mathbb{Z}\left[\mathfrak{t}_{r}\right]$-graded subspace (here and below $\mathfrak{H}$ is a subalgebra of $W(n)$ containing $\mathfrak{t}_{r}$ ).

- $\mathfrak{H}_{\alpha}^{\left[\mathfrak{t}_{r}\right]}: \mathfrak{t}_{r}$-graded subspace (root subspace).

- $\mathfrak{H}_{[i]}$ : standard graded subspace (associated with $\mathfrak{t}_{0}$ ), where $\mathfrak{H}$ contains $\mathfrak{t}_{0}$.

For Borel subalgebras:

- b: the standard Borel subalgebra in $W_{[0]} \cong \mathfrak{g l}(n)$ (see $\left.\S 2.5\right)$.

- $L^{(i)}$ for a Lie algebra $L$ : the $i$-th derived ideal (see $\left.\S 2.5\right)$.

- $\mathrm{B}_{q}$ : Borel subspaces of $W(n), q=0,1, \ldots, n$ (see $\S 3.1$ ). All of them are finally proved to be Borel subalgebras and to parameterize the iso-classes of homogeneous Borel subalgebras of $W(n)$.

- $\mathrm{B}_{0}$ and $\mathrm{B}_{n}$ : the unswitched Borel subspace and the fully-switched Borel subspace of $W(n)$, respectively (see $\S 3.1$ ).

- $\mathrm{B}_{0}\left(u_{1}, \ldots, u_{q}\right)$ and $\mathrm{B}_{q}\left(u_{1}, \ldots, u_{q}\right)$ : the same meaning as above with respect to $u_{1}, \ldots, u_{q}$ (see Conventions 3.1).

- $r(\mathcal{B})$ : an invariant of homogeneous Borel conjugacy classes (see $§ 4.1$ ).

\section{Acknowledgments}

The author thanks Hao Chang, Rolf Farnsteiner, Zongzhu Lin, Ke Ou, Xin Wen, Husileng Xiao, Yufeng Yao and Mengmeng Zhang for helpful dis- 
cussions. The author is very grateful to Shun-Jen Cheng and the anonymous referees for their valuable comments on the manuscript.

\section{References}

1. R. Bezrukavnikov, I. Mirković and D. Rumynin, Localization of modules for a semisimple Lie algebra in prime characteristic, Annals of Math., 167 (2008), 945-991.

2. J.-M. Bois, R. Farnsteiner and B. Shu, Weyl groups for non-classical restricted Lie algebras and the Chevalley restriction theorem, Forum Math., 26 (2014), 1333-1379.

3. S. P. Demuškin, Cartan subalgebras of the simple Lie $p$-algebras $W_{n}$ and $S_{n}$, Siberian Math. J., 11 (1970), 233-245.

4. S. P. Demuškin, Cartan subalgebras of simple nonclassical Lie $p$-algebras, Math. USSR Izv., 6 (1972), 905-924.

5. R. Farnsteiner, Varieties of tori and Cartan subalgebras of restricted Lie algebras, Trans. Amer. Math. Soc., 356 (2004), 4181-4236.

6. R. Hartshorne, Algebraic Geometry, Springer-Verlag, New York, 1977.

7. S. Herpel and D. I. Stewart, On the smoothness of normalisers and the subalgebra structure of modular Lie algebras, arXiv:1402.6280v1 [math.GR].

8. J. E. Humphreys, Algebraic groups and modular Lie algebras, Memoirs A. M. S. (71), Amer. Math. Soc., Providence, PI, 1967.

9. J. E. Humphreys, Linear Algebraic Groups, Springer-Verlag, New York, 1981.

10. J. C. Jantzen, Representations of algebraic groups, second edition, Amer. Math. Soc., Providence, PI, 2003.

11. J. C. Jantzen, Nilpotent orbits in representation theory, in "Lie Theory" PM 228, Birkhäuser Boston, 2004, 1-206.

12. K. Ou, Weyl groups and Geometric setting of Lie algebras of Cartan type, Ph. D thesis, East China Normal University, 2016.

13. K. Ou, B. Shu and H. Xiao, Generic property and conjugacy classes of homogeneous Borel subalgebras of restricted Lie algebras of Cartan type (II), in preparation.

14. A. A. Premet, On Cartan subalgebras of Lie p-algebras, Math. USSR Izv., 29 (1987), 145-157.

15. A. A. Premet, Regular Cartan subalgebras and nilpotent elements in restricted Lie algebras, Math. USSR Sb., 66 (1989), 555-570.

16. A. A. Premet, The theorem on restriction of invariants and nilpotent elements in $W_{n}$, Math. USSR Sb., 73 (1992), 135-159.

17. A. A. Premet and H. Strade, Classification of finite dimensional simple Lie algebras in prime characteristics, Representations of algebraic groups, quantum groups, and Lie algebras, 185-214, Contemp. Math., 413, Amer. Math. Soc., Providence, RI, 2006. 
18. G. B. Seligman, Modular Lie Algebras, Springer-Verlag Berlin Heidelberg, 1967.

19. H. Strade, Simple Lie Algebras over Fields of Positive Charactersitic I. Structure Theory, Walter de Gruyter, Berlin, 2004.

20. H. Strade and R. Farnsteiner, Modular Lie Algebras and Their Representations, Marcel Dekker, New York, 1988.

21. R. L. Wilson, Automorphisms of graded Lie algebras of Cartan type, Comm. Algebra, 3 (1975), 591-613.

22. Y. Yao and H. Chang, Borel subalgebras of the Witt algebra $W_{1}$, arXiv:1212.4349 [MathRT]. 